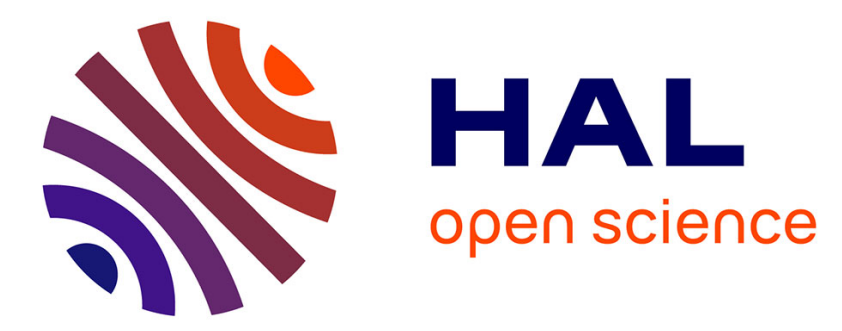

\title{
Présentation des premiers éléments du diagnostic territorial de L'AMETRA Montpellier
}

\author{
Baris Celik
}

\section{To cite this version:}

Baris Celik. Présentation des premiers éléments du diagnostic territorial de L'AMETRA Montpellier. Médecine humaine et pathologie. 2018. dumas-02956266

\section{HAL Id: dumas-02956266 https://dumas.ccsd.cnrs.fr/dumas-02956266}

Submitted on 2 Oct 2020

HAL is a multi-disciplinary open access archive for the deposit and dissemination of scientific research documents, whether they are published or not. The documents may come from teaching and research institutions in France or abroad, or from public or private research centers.
L'archive ouverte pluridisciplinaire HAL, est destinée au dépôt et à la diffusion de documents scientifiques de niveau recherche, publiés ou non, émanant des établissements d'enseignement et de recherche français ou étrangers, des laboratoires publics ou privés.

\section{(1)(1) $\$(0)$}

Distributed under a Creative Commons Attribution - NonCommercial - ShareAlikel 4.0 


\section{UNIVERSITE DE MONTPELLIER \\ FACULTE DE MEDECINE MONTPELLIER-NIMES}

\section{THESE}

Pour obtenir le titre de

DOCTEUR EN MEDECINE

Présentée et soutenue publiquement par

Baris CELIK

Le $26 / 10 / 2018$

\section{PRESENTATION DES PREMIERS ELEMENTS DU DIAGNOSTIC TERRITORIAL DE} L'AMETRA MONTPELLIER

Directeur de thèse : Docteur Cyril BERNARDET

\section{JURY}

Président : Professeur Philippe COURTET

Assesseurs (y compris directeur de thèse) :

Docteur Cyril BERNARDET*

Professeur Pierre DUJOLS

MCU-PH François-Xavier LESAGE 


\section{UNIVERSITE DE MONTPELLIER}

FACULTE DE MEDECINE MONTPELLIER-NIMES

\section{THESE}

Pour obtenir le titre de

DOCTEUR EN MEDECINE

Présentée et soutenue publiquement par

Baris CELIK

Le $26 / 10 / 2018$

\section{PRESENTATION DES PREMIERS ELEMENTS DU DIAGNOSTIC TERRITORIAL DE L'AMETRA MONTPELLIER}

Directeur de thèse : Docteur Cyril BERNARDET

JURY

Président : Professeur Philippe COURTET

Assesseurs (y compris directeur de thèse) :

Docteur Cyril BERNARDET*

Professeur Pierre DUJOLS

MCU-PH François-Xavier LESAGE 
ANNEE UNIVERSITAIRE 2017 - 2018

\section{PERSONNEL ENSEIGNANT}

\section{Professeurs Honoraires}

ALLIEU Yves

ALRIC Robert

ARNAUD Bernard

ASTRUC Jacques

AUSSILLOUX Charles

AVEROUS Michel

AYRAL Guy

BAILLAT Xavier

BALDET Pierre

BALDY-MOULINIER Michel

BALMES Jean-Louis

BALMES Pierre

BANSARD Nicole

BAYLET René

BILLIARD Michel

BLARD Jean-Marie

BLAYAC Jean Pierre

BLOTMAN Francis

BONNEL François

BOUDET Charles

BOURGEOIS Jean-Marie

BRUEL Jean Michel

BUREAU Jean-Paul

BRUNEL Michel

CALLIS Albert

CANAUD Bernard

CASTELNAU Didier

CHAPTAL Paul-André

CIURANA Albert-Jean

CLOT Jacques

D'ATHIS Françoise

DEMAILLE Jacques

DESCOMPS Bernard

\section{Professeurs Emérites}

ARTUS Jean-Claude

BLANC François

BOULENGER Jean-Philippe

BOURREL Gérard

BRINGER Jacques

CLAUSTRES Mireille

DAURES Jean-Pierre

DAUZAT Michel

DEDET Jean-Pierre

ELEDJAM Jean-Jacques

GUERRIER Bernard

JOURDAN Jacques

MAURY Michèle

MILLAT Bertrand
DIMEGLIO Alain

DU CAILAR Jacques

DUBOIS Jean Bernard

DUMAS Robert

DUMAZER Romain

ECHENNE Bernard

FABRE Serge

FREREBEAU Philippe

GALIFER René Benoît

GODLEWSKI Guilhem

GRASSET Daniel

GROLLEAU-RAOUX Robert

GUILHOU Jean-Jacques

HERTAULT Jean

HUMEAU Claude

JAFFIOL Claude

JANBON Charles

JANBON François

JARRY Daniel

JOYEUX Henri

LAFFARGUE François

LALLEMANT Jean Gabriel

LAMARQUE Jean-Louis

LAPEYRIE Henri

LESBROS Daniel

LOPEZ François Michel

LORIOT Jean

LOUBATIERES Marie

Madeleine

MAGNAN DE BORNIER

Bernard

MARY Henri

MATHIEU-DAUDE Pierre
MEYNADIER Jean

MICHEL François-Bernard

MICHEL Henri

MION Charles

MION Henri

MIRO Luis

NAVARRO Maurice

NAVRATIL Henri

OTHONIEL Jacques

PAGES Michel

PEGURET Claude

POUGET Régis

PUECH Paul

PUJOL Henri

PUJOL Rémy

RABISCHONG Pierre

RAMUZ Michel

RIEU Daniel

RIOUX Jean-Antoine

ROCHEFORT Henri

ROUANET DE VIGNE LAVIT

Jean Pierre

SAINT AUBERT Bernard

SANCHO-GARNIER Hélène

SANY Jacques

SENAC Jean-Paul

SERRE Arlette

SIMON Lucien

SOLASSOL Claude

THEVENET André

VIDAL Jacques

VISIER Jean Pierre
MARES Pierre

MONNIER Louis

PRAT Dominique

PRATLONG Francine

PREFAUT Christian

PUJOL Rémy

ROSSI Michel

SULTAN Charles

TOUCHON Jacques

VOISIN Michel

ZANCA Michel 


\section{Professeurs des Universités - Praticiens Hospitaliers}

\section{PU-PH de classe exceptionnelle}

ALBAT Bernard - Chirurgie thoracique et cardiovasculaire

ALRIC Pierre - Chirurgie vasculaire ; médecine vasculaire (option chirurgie vasculaire)

BACCINO Eric - Médecine légale et droit de la santé

BASTIEN Patrick - Parasitologie et mycologie

BONAFE Alain - Radiologie et imagerie médicale

CAPDEVILA Xavier - Anesthésiologie-réanimation

COMBE Bernard - Rhumatologie

COSTA Pierre - Urologie

COTTALORDA Jérôme - Chirurgie infantile

COUBES Philippe - Neurochirurgie

CRAMPETTE Louis - Oto-rhino-laryngologie

CRISTOL Jean Paul - Biochimie et biologie moléculaire

DAVY Jean Marc - Cardiologie

DE LA COUSSAYE Jean Emmanuel - Anesthésiologie-réanimation

DELAPORTE Eric - Maladies infectieuses ; maladies tropicales

DE WAZIERES Benoît - Médecine interne ; gériatrie et biologie du vieillissement, médecine

générale, addictologie

DOMERGUE Jacques - Chirurgie générale

DUFFAU Hugues - Neurochirurgie

DUJOLS Pierre - Biostatistiques, informatique médicale et technologies de la communication

ELIAOU Jean François - Immunologie

FABRE Jean Michel - Chirurgie générale

GUILLOT Bernard - Dermato-vénéréologie

HAMAMAH Samir-Biologie et Médecine du développement et de la reproduction ; gynécologie médicale

HEDON Bernard-Gynécologie-obstétrique ; gynécologie médicale

HERISSON Christian-Médecine physique et de réadaptation

JABER Samir-Anesthésiologie-réanimation

JEANDEL Claude-Médecine interne ; gériatrie et biologie du vieillissement, médecine générale, addictologie

JONQUET Olivier-Réanimation ; médecine d'urgence

JORGENSEN Christian-Thérapeutique ; médecine d'urgence ; addictologie

KOTZKI Pierre Olivier-Biophysique et médecine nucléaire

LANDAIS Paul-Epidémiologie, Economie de la santé et Prévention

LARREY Dominique-Gastroentérologie ; hépatologie ; addictologie

LEFRANT Jean-Yves-Anesthésiologie-réanimation

LE QUELLEC Alain-Médecine interne ; gériatrie et biologie du vieillissement, médecine générale, addictologie

MARTY-ANE Charles - Chirurgie thoracique et cardiovasculaire

MAUDELONDE Thierry - Biologie cellulaire

MERCIER Jacques - Physiologie

MESSNER Patrick - Cardiologie

MOURAD Georges-Néphrologie

PELISSIER Jacques-Médecine physique et de réadaptation

RENARD Eric-Endocrinologie, diabète et maladies métaboliques ; gynécologie médicale

REYNES Jacques-Maladies infectieuses, maladies tropicales

RIBSTEIN Jean-Médecine interne ; gériatrie et biologie du vieillissement, médecine générale,

addictologie

RIPART Jacques-Anesthésiologie-réanimation

ROUANET Philippe-Cancérologie ; radiothérapie

SCHVED Jean François-Hématologie; Transfusion

TAOUREL Patrice-Radiologie et imagerie médicale

UZIEL Alain -Oto-rhino-laryngologie

VANDE PERRE Philippe-Bactériologie-virologie ; hygiène hospitalière

YCHOU Marc-Cancérologie ; radiothérapie

PU-PH de $1^{\text {re }}$ classe

AGUILAR MARTINEZ Patricia-Hématologie ; transfusion 
AVIGNON Antoine-Nutrition

AZRIA David -Cancérologie ; radiothérapie

BAGHDADLI Amaria-Pédopsychiatrie ; addictologie

BEREGI Jean-Paul-Radiologie et imagerie médicale

BLAIN Hubert-Médecine interne ; gériatrie et biologie du vieillissement, médecine générale, addictologie

BLANC Pierre-Gastroentérologie ; hépatologie ; addictologie

BORIE Frédéric-Chirurgie digestive

BOULOT Pierre-Gynécologie-obstétrique ; gynécologie médicale

CAMBONIE Gilles -Pédiatrie

CAMU William-Neurologie

CANOVAS François-Anatomie

CARTRON Guillaume-Hématologie ; transfusion

CHAMMAS Michel-Chirurgie orthopédique et traumatologique

COLSON Pascal-Anesthésiologie-réanimation

CORBEAU Pierre-Immunologie

COSTES Valérie-Anatomie et cytologie pathologiques

COURTET Philippe-Psychiatrie d'adultes ; addictologie

CYTEVAL Catherine-Radiologie et imagerie médicale

DADURE Christophe-Anesthésiologie-réanimation

DAUVILLIERS Yves-Physiologie

DE TAYRAC Renaud-Gynécologie-obstétrique, gynécologie médicale

DEMARIA Roland-Chirurgie thoracique et cardio-vasculaire

DEMOLY Pascal-Pneumologie ; addictologie

DEREURE Olivier-Dermatologie - vénéréologie

DROUPY Stéphane -Urologie

DUCROS Anne-Neurologie -

FRAPIER Jean-Marc-Chirurgie thoracique et cardiovasculaire

KLOUCHE Kada-Réanimation ; médecine d'urgence

KOENIG Michel-Génétique moléculaire

LABAUGE Pierre- Neurologie

LAFFONT Isabelle-Médecine physique et de réadaptation

LAVABRE-BERTRAND Thierry-Cytologie et histologie

LECLERCQ Florence-Cardiologie

LEHMANN Sylvain-Biochimie et biologie moléculaire

LUMBROSO Serge-Biochimie et Biologie moléculaire

MARIANO-GOULART Denis-Biophysique et médecine nucléaire

MATECKI Stéfan -Physiologie

MEUNIER Laurent-Dermato-vénéréologie

MONDAIN Michel-Oto-rhino-laryngologie

MORIN Denis-Pédiatrie

NAVARRO Francis-Chirurgie générale

PAGEAUX Georges-Philippe-Gastroentérologie ; hépatologie ; addictologie

PETIT Pierre-Pharmacologie fondamentale ; pharmacologie clinique ; addictologie

PERNEY Pascal-Médecine interne ; gériatrie et biologie du vieillissement, médecine générale, addictologie

PUJOL Jean Louis-Pneumologie ; addictologie

PUJOL Pascal-Biologie cellulaire

PURPER-OUAKIL Diane-Pédopsychiatrie ; addictologie

QUERE Isabelle-Chirurgie vasculaire ; médecine vasculaire (option médecine vasculaire)

SOTTO Albert-Maladies infectieuses ; maladies tropicales

TOUITOU Isabelle-Génétique

TRAN Tu-Anh-Pédiatrie

VERNHET Hélène-Radiologie et imagerie médicale

\section{PU-PH de 2ème classe}

ASSENAT Éric-Gastroentérologie ; hépatologie ; addictologie

BERTHET Jean-Philippe-Chirurgie thoracique et cardiovasculaire

BOURDIN Arnaud-Pneumologie ; addictologie

CANAUD Ludovic-Chirurgie vasculaire ; Médecine Vasculaire

CAPDEVIELLE Delphine-Psychiatrie d'Adultes ; addictologie

CAPTIER Guillaume-Anatomie 
CAYLA Guillaume-Cardiologie

CHANQUES Gérald-Anesthésiologie-réanimation

COLOMBO Pierre-Emmanuel-Cancérologie ; radiothérapie

COSTALAT Vincent-Radiologie et imagerie médicale

COULET Bertrand-Chirurgie orthopédique et traumatologique

CUVILLON Philippe-Anesthésiologie-réanimation

DAIEN Vincent-Ophtalmologie

DE VOS John-Cytologie et histologie

DORANDEU Anne-Médecine légale -

DUPEYRON Arnaud-Médecine physique et de réadaptation

FESLER Pierre-Médecine interne ; gériatrie et biologie du vieillissement, médecine générale, addictologie

GARREL Renaud -Oto-rhino-laryngologie

GAUJOUX Viala Cécile-Rhumatologie

GENEVIEVE David-Génétique

GODREUIL Sylvain-Bactériologie-virologie ; hygiène hospitalière

GUILLAUME Sébastien-Urgences et Post urgences psychiatriques -

GUILPAIN Philippe-Médecine Interne, gériatrie et biologie du vieillissement; addictologie

GUIU Boris-Radiologie et imagerie médicale

HAYOT Maurice-Physiologie

HOUEDE Nadine-Cancérologie ; radiothérapie

JACOT William-Cancérologie ; Radiothérapie

JUNG Boris-Réanimation ; médecine d'urgence

KALFA Nicolas-Chirurgie infantile

KOUYOUMDJIAN Pascal-Chirurgie orthopédique et traumatologique

LACHAUD Laurence-Parasitologie et mycologie

LALLEMANT Benjamin-Oto-rhino-laryngologie

LAVIGNE Jean-Philippe-Bactériologie-virologie ; hygiène hospitalière

LE MOING Vincent-Maladies infectieuses ; maladies tropicales

LETOUZEY Vincent-Gynécologie-obstétrique ; gynécologie médicale

LOPEZ CASTROMAN Jorge-Psychiatrie d'Adultes ; addictologie

LUKAS Cédric-Rhumatologie

MAURY Philippe-Chirurgie orthopédique et traumatologique

MILLET Ingrid-Radiologie et imagerie médicale

MORANNE Olvier-Néphrologie

MOREL Jacques -Rhumatologie

NAGOT Nicolas-Biostatistiques, informatique médicale et technologies de la communication

NOCCA David-Chirurgie digestive

PANARO Fabrizio-Chirurgie générale

PARIS Françoise-Biologie et médecine du développement et de la reproduction ; gynécologie médicale

PASQUIE Jean-Luc-Cardiologie

PEREZ MARTIN Antonia-Physiologie

POUDEROUX Philippe-Gastroentérologie ; hépatologie ; addictologie

PRUDHOMME Michel-Anatomie

RIGAU Valérie-Anatomie et cytologie pathologiques

RIVIER François-Pédiatrie

ROGER Pascal-Anatomie et cytologie pathologiques

ROSSI Jean François-Hématologie ; transfusion

ROUBILLE François-Cardiologie

SEBBANE Mustapha-Anesthésiologie-réanimation

SEGNARBIEUX François-Neurochirurgie

SIRVENT Nicolas-Pédiatrie

SOLASSOL Jérôme-Biologie cellulaire

SULTAN Ariane-Nutrition

THOUVENOT Éric-Neurologie

THURET Rodolphe-Urologie

VENAIL Frédéric-Oto-rhino-laryngologie

VILLAIN Max-Ophtalmologie

VINCENT Denis -Médecine interne ; gériatrie et biologie du vieillissement, médecine générale, addictologie

VINCENT Thierry-Immunologie 


\section{PROFESSEURS DES UNIVERSITES}

$1^{\text {re }}$ classe :

COLINGE Jacques - Cancérologie, Signalisation cellulaire et systèmes complexes

$2^{\mathrm{ème}}$ classe :

LAOUDJ CHENIVESSE Dalila - Biochimie et biologie moléculaire

VISIER Laurent - Sociologie, démographie

PROFESSEURS DES UNIVERSITES - Médecine générale

$1^{\text {re }}$ classe :

LAMBERT Philippe

2ème classe :

AMOUYAL Michel

DAVID Michel

PROFESSEURS ASSOCIES - Médecine Générale

RAMBAUD Jacques

PROFESSEURS ASSOCIES - Médecine

BESSIS Didier - Dermato-vénéréologie)

PERRIGAULT Pierre-François - Anesthésiologie-réanimation ; médecine d'urgence

ROUBERTIE Agathe - Pédiatrie

\section{Maîtres de Conférences des Universités - Praticiens Hospitaliers}

\section{MCU-PH Hors classe}

CACHEUX-RATABOUL Valère-Génétique

CARRIERE Christian-Bactériologie-virologie ; hygiène hospitalière

CHARACHON Sylvie-Bactériologie-virologie ; hygiène hospitalière

FABBRO-PERAY Pascale-Epidémiologie, économie de la santé et prévention

HILLAIRE-BUYS Dominique-Pharmacologie fondamentale ; pharmacologie clinique ; addictologie

PELLESTOR Franck-Cytologie et histologie

PUJOL Joseph-Anatomie

RAMOS Jeanne-Anatomie et cytologie pathologiques

RICHARD Bruno-Thérapeutique ; addictologie

RISPAIL Philippe-Parasitologie et mycologie

SEGONDY Michel-Bactériologie-virologie ; hygiène hospitalière

STOEBNER Pierre -Dermato-vénéréologie

MCU-PH de $1^{\text {re }}$ classe

ALLARDET-SERVENT Annick-Bactériologie-virologie; hygiène hospitalière

BADIOU Stéphanie-Biochimie et biologie moléculaire

BOUDOUSQ Vincent-Biophysique et médecine nucléaire

BOULLE Nathalie-Biologie cellulaire

BOURGIER Céline-Cancérologie ; Radiothérapie

BRET Caroline -Hématologie biologique

COSSEE Mireille-Génétique Moléculaire

GABELLE DELOUSTAL Audrey-Neurologie

GIANSILY-BLAIZOT Muriel-Hématologie ; transfusion

GIRARDET-BESSIS Anne-Biochimie et biologie moléculaire

LAVIGNE Géraldine-Hématologie ; transfusion

LE QUINTREC Moglie-Néphrologie

MATHIEU Olivier-Pharmacologie fondamentale ; pharmacologie clinique ; addictologie

MENJOT de CHAMPFLEUR Nicolas-Neuroradiologie 
MOUZAT Kévin-Biochimie et biologie moléculaire

PANABIERES Catherine-Biologie cellulaire

PHILIBERT Pascal-Biologie et médecine du développement et de la reproduction

RAVEL Christophe - Parasitologie et mycologie

SCHUSTER-BECK Iris-Physiologie

STERKERS Yvon-Parasitologie et mycologie

TUAILLON Edouard-Bactériologie-virologie ; hygiène hospitalière

YACHOUH Jacques-Chirurgie maxillo-faciale et stomatologie

MCU-PH de $2^{\text {éme }}$ classe

BERTRAND Martin-Anatomie

BRUN Michel-Bactériologie-virologie ; hygiène hospitalière

DU THANH Aurélie-Dermato-vénéréologie

GALANAUD Jean Philippe-Médecine Vasculaire

GOUZI Farès-Physiologie

JEZIORSKI Éric-Pédiatrie

KUSTER Nils-Biochimie et biologie moléculaire

LESAGE François-Xavier-Médecine et Santé au Travail

MAKINSON Alain-Maladies infectieuses, Maladies tropicales

MURA Thibault-Biostatistiques, informatique médicale et technologies de la communication

OLIE Emilie-Psychiatrie d'adultes ; addictologie

THEVENIN-RENE Céline-Immunologie

\title{
MAITRES DE CONFERENCES DES UNIVERSITES - Médecine Générale COSTA David \\ FOLCO-LOGNOS Béatrice
}

\author{
MAITRES DE CONFERENCES ASSOCIES - Médecine Générale \\ CLARY Bernard \\ GARCIA Marc \\ MILLION Elodie \\ PAVAGEAU Sylvain \\ REBOUL Marie-Catherine \\ SEGURET Pierre
}

\section{MAITRES DE CONFERENCES DES UNIVERSITES}

Maîtres de Conférences hors classe

BADIA Eric - Sciences biologiques fondamentales et cliniques

\author{
Maîtres de Conférences de classe normale \\ BECAMEL Carine - Neurosciences \\ BERNEX Florence - Physiologie \\ CHAUMONT-DUBEL Séverine - Sciences du médicament et des autres produits de santé \\ CHAZAL Nathalie - Biologie cellulaire \\ DELABY Constance - Biochimie et biologie moléculaire \\ GUGLIELMI Laurence - Sciences biologiques fondamentales et cliniques \\ HENRY Laurent - Sciences biologiques fondamentales et cliniques \\ LADRET Véronique - Mathématiques appliquées et applications des mathématiques \\ LAINE Sébastien - Sciences du Médicament et autres produits de santé \\ LE GALLIC Lionel - Sciences du médicament et autres produits de santé \\ LOZZA Catherine - Sciences physico-chimiques et technologies pharmaceutiques \\ MAIMOUN Laurent - Sciences physico-chimiques et ingénierie appliquée à la santé \\ MOREAUX Jérôme - Science biologiques, fondamentales et cliniques \\ MORITZ-GASSER Sylvie - Neurosciences \\ MOUTOT Gilles - Philosophie \\ PASSERIEUX Emilie - Physiologie \\ RAMIREZ Jean-Marie - Histologie \\ TAULAN Magali - Biologie Cellulaire
}

\section{PRATICIENS HOSPITALIERS UNIVERSITAIRES}


BASTIDE Sophie-Epidémiologie, économie de la santé et prévention

FAILLIE Jean-Luc-

Pharmacologie fondamentale ; pharmacologie clinique ; addictologie

GATINOIS Vincent-Histologie, embryologie et cytogénétique

HERLIN Christian -Chirurgie plastique ; reconstructrice et esthétique ; brûlologie

HERRERO Astrid-Chirurgie générale

PANTEL Alix-Bactériologie-virologie ; hygiène hospitalière

PERS Yves-Marie-Thérapeutique, médecine d'urgence ; addictologie

PINETON DE CHAMBRUN Guillaume-Gastroentérologie ; hépatologie ; addictologie

TORRE Antoine-Gynécologie-obstétrique ; gynécologie médicale 


\section{REMERCIEMENTS}

Au Professeur COURTET, c'est un grand honneur de vous avoir comme Président du Jury de thèse. Je vous suis profondément reconnaissant d'avoir accordé votre confiance à ce travail. Merci également d'avoir accepté d'accueillir des internes en médecine du travail dans vos services et d'avoir rendu la psychiatrie aussi attractive et passionnante. Je vous prie de trouver ici l'expression de mes sincères remerciements et de ma haute considération.

Au Professeur DUJOLS, merci d'avoir accepté de juger ce travail de thèse, merci pour le temps accordé au jugement de mon travail. Soyez assuré de mon respect sincère.

Au Dr LESAGE, pour avoir encadré notre filière pendant ces 4 années et de répondre toujours présent lorsque nous avons des questions à poser. Merci pour tout ce que tu m'as appris. Merci de m'avoir toujours encouragé et énormément aidé pour ce travail de thèse.

Au Dr Cyril BERNARDET, pour son soutien inconditionnel, sa gentillesse, sa présence et sa bienveillance à mon égard. J'ai appris énormément à tes cotés et tu m'as toujours fait confiance et su me remotiver. Merci de $m$ 'avoir encadré pour cette thèse. Je te verrai encore quelques temps comme mon tuteur si tu ne m'en veux pas, mais je te vois surtout comme un ami. En tout cas tu es vraiment une personne géniale et c'est un plaisir de travailler à tes côtés, j'espère que nous partagerons ce plaisir encore longtemps.

A mes parents, que j'espère avoir rendu fier (Anne, Baba, umarimki benimle gurur duyuyorsunuzdur. Sizlere cok ama cok minettarim, bana sonsuzca guvenip desteklediginiz icin, bana verdiginiz sevgi icin. Slzleri cok seviyorum. Artik uzakta olsamda, her zaman yaninizdayim).

A mon petit frère et ma petite sœur que j'aime plus que tout et qui me rendent chaque jour fier d'eux.

Il y a eu du changement sur ces 4 dernières années, j'ai rencontré des gens aux grands cœurs en plus des gens exceptionnels que je connaissais déjà.

Je voudrais tout particulièrement remercier celle qui a tout chambouler depuis ces deux dernières années, celle qu'aujourd'hui je peux appeler ma moitié, celle qui me fait avancer. Grâce à toi je peux dire que je n'ai jamais été aussi heureux de ma vie. Tu es mon plus beau cadeau au quotidien. Merci de me soutenir quoi qu'il arrive, de me donner autant d'amour, de me faire rire. Sache que tu occupes la place centrale dans tous mes rêves d'avenir et mes projets futurs. Je nous souhaite que du bonheur ensemble et espère un jour avoir l'occasion de t'appeler ma femme. Merci pour tout mon Doudou.

Aucun ordre de préférence dans ces remerciement, car je les vois venir les pinailleurs. Tout le monde a sa place dans mon cœur.

A Davy mon acolyte depuis la 6eme. Ca a matché depuis le premier jour où je me suis assis à côté de ce grand maigre à lunettes. Ca fait maintenant 18ans que ça colle entre nous et pas 
de raisons pour que cela ne continue pas. J'espère vraiment que tu nous reviendras rapidement dans le sud.

A Thomas, l'un des hommes au plus grand cœur que je connaisse, merci pour tout, merci de m'avoir fait venir à Montpellier. Sans toi je serais probablement à Bordeaux en train de surfer la mèche au vent. Je te dois en partie ce qui m'arrive et je ne l'oublie pas. Reste comme tu es.

A ma turkish Family, Reco, Ozgur, Umut, Serdar, Mehmet. Vous êtes mes piliers, la base de la base. Sans vous la P1 ne serait jamais passée. A toutes nos soirées, si vous aviez fait médecine avec moi, vous seriez en P10 ( (); ). Mention spéciale à Reco, I'homme auquel j'aimerai ressembler dans sa générosité et sa bonté. Et à Ozgur pour toutes nos soirées en duo, à nos longues discussions dans la voiture les soirs d'hiver, merci d'avoir toujours trouvé les bons mots pour m'encourager. Merci à tous les frères.

J'aurai aimé partager avec chacun mon esprit de poète et tous vous accordez une page, mais je vais aller assez vite.

Merci à toute la team Saint Eloi ;

A Taki +++ pour son aide précieuse et pour me casser les jambes au foot.

A Nath pour sa bonne humeur éternelle et qui sait toujours redonner le sourire.

A Suzanne pour sa mauvaise humeur éternelle (que j'adore) mais ça c'est ton coté chirurgien, en vrai je sais que tu es pleine d'amour.

À Guillaume pour tous ses coups de cœur que j'aime écouter.

A Boran le meilleur coloc qui soit et avec qui, on n'a jamais réussi à regarder un film sans qu'il ne s'endorme.

A Quentin pour sa générosité et avec qui on a rêvé d'être musclé, un jour on fera business ensemble je ne $m^{\prime}$ inquiète pas.

A Sarah pour toujours rigoler à toutes mes blagues.

A Antonia pour sa capacité à trancher des que nous ne savons pas quoi faire.

A Victoria pour ne pas avoir réussi à me mettre à la Salsa.

A Pauline la bisounours pour tout l'amour qu'elle donne au groupe.

A Hamza mon gars sur pour sa capacité à me faire dépenser.

A Emilie pour être capable de nous suivre dans n'importe quel plan (\#SoiréeCarca).

A Stan pour toujours réussir à nous faire faire ce qu'il a envi.

A Nina pour être forte en charriage photo

Sans oublier, Kevin, Pappalardo, Simon, Sylvain, Aurelie la crazy danseuse.

Mention à Redha et Alesso que je connaissais mais que j'ai appris à connaitre vraiment à Montpellier, c'est un plaisir de vous voir à chaque fois les gars.

A Verchère notre voisin sudiste qui vient nous voir régulièrement et qui nous accueille à Marseille.

A Mouss, meilleur dealer de MPG et ex champion de PL.

A Anis, que je connais depuis la maternelle, compagnon de P1 et bien plus encore, et que je considère énormément. 
A Wafa pour nos nombreuses soirées à bosser très tard.

A mes futurs confrères parisiens Nash et Achilus.

A Ouaoua et Dallal mes arabicas préférées que j'espère voir plus souvent.

A Hubi et Baptiste, et oui le foot peut créer de vraies amitiés. Mais aussi à tous les autres potes du foot, Sam, Vic, Ade qui me permettent de perdre toutes les semaines et de me faire redescendre sur terre.

Merci à tous mes cointernes qui m'ont fait passer de bons moments en stage, Emmanuelle, Dimitri, Sofiene, Marie, Farah.

Merci à mes futurs confrères que j'adore même si je ne suis pas souvent présent au réunion, Liza, Flo, Soraya et mention spéciale à Guigui qui est toujours présent en cas de besoin et pour réunir le groupe et à Nico pour avoir géré tous nos stages.

Un énorme merci à Bruno pour avoir toujours pris le temps de m'aider et sans qui ce travail n'aurait pas vu le jour.

A Aline et Evelyne, mes coups de cœur de l'Ametra. Merci à Mme DEMON de m'accorder sa confiance.

A Caroline, JF et Marie Thé, de m'avoir accueilli et de m'avoir fait rapidement sentir à l'aise.

A tous les amis de la fac qui ont rendu ces années meilleures, Amina, Elodie, Sonia, Nor, Bende, Benji, Kelly...

A tous les amis du lycée, Romain, Bruno, Clément...

MERCI A TOUS d'avoir fait de moi ce que je suis aujourd'hui. 


\section{Table des matières}

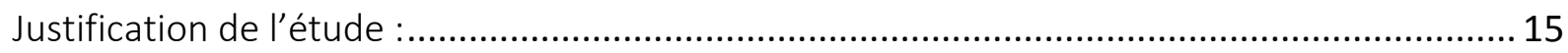

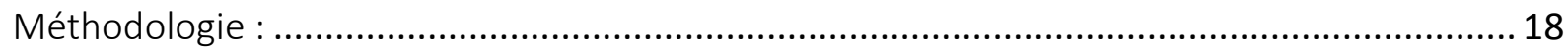

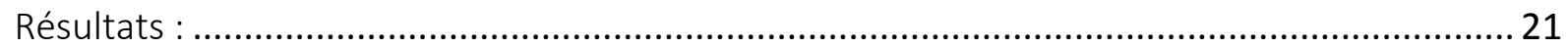

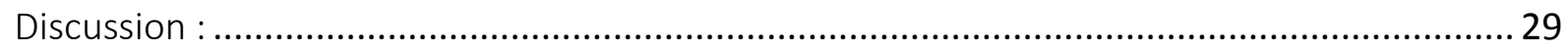

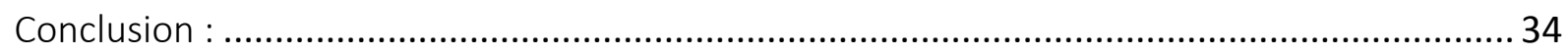

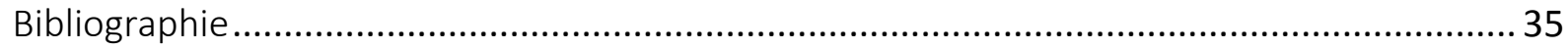

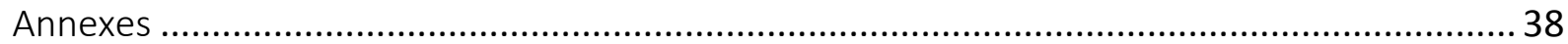

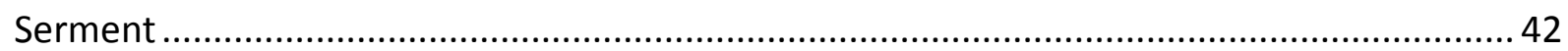

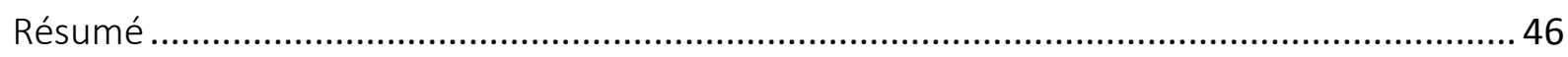




\section{Justification de l'étude :}

Actuellement en France, le système de santé dispose de nombreuses données regroupées au sein du Système National des Données de Santé (SNDS). Ces données sont issues de diverses sources telles que l'assurance maladie (le système national d'information interrégime de I'assurance maladie, SNIIRAM)(1), le Programme de Médicalisation des systèmes d'information (PMSI) qui recueille les données des hôpitaux ou encore le Recueil d'Information Médicalisé en Psychiatrie, le RIM P(2). Le SNDS est un système unique en Europe et constitue une avancée considérable pour analyser et améliorer la santé de la population.

Par contre, le système de Santé au Travail ne dispose que de peu d'outils de collecte de données. Les principaux systèmes sont d'une part les données d'accidents du travail (AT) et maladies professionnelles (MP) issues des caisses d'assurance maladie, l'observatoire de veille sanitaire national, Evolution et Relation En Santé Travail (EVREST)(3) qui a progressivement été mis en place depuis 2007 avec un réseau de médecins du travail exerçant dans des services de santé au travail, et le réseau national de vigilance et de prévention des pathologies professionnelles (RNV3P), collectant les données issues des centres de consultations de pathologies professionnelles, permettant d'observer l'émergence(4) de nouvelles pathologies. L'analyse de ces indicateurs donne régulièrement lieux à des rapports régionaux(5,6). Mais sans l'aide d'organismes spécialisés tels que la Caisse d'Assurance Retraite et de la Santé au Travail (CARSAT) ou la Caisse Primaire d'Assurance Maladie (CPAM), il reste difficile pour les services de santé au travail de recueillir des informations exhaustives et interprétables.

En dépit d'une généralisation des dossiers médicaux de santé au travail informatisés, et d'un large maillage de la population salariée sur le territoire, il n'y a pas de collecte des données issue du système de santé au travail, pourtant essentiel au pilotage des stratégies de prévention en santé au travail. Cela devient également une des priorités du gouvernement. Dans son rapport en réponse à la lettre de mission du premier ministre (annexe 1), la députée Mme LECOCQ recommande (annexe 2) notamment la mise en place d'outils permettant « l'exploitation collective de données ". 
L'informatisation des dossiers médicaux de santé au travail recommandée par la Haute Autorité de Santé (HAS)(5), le Conseil National de l'Ordre des Médecins (CNOM) et l'utilisation d'un thésaurus harmonisé enrichissent les systèmes d'informations propres à chaque service de santé au travail. Or, la grande majorité de ces données ne sont ni extraites, ni statistiquement analysées à ce jour.

Promouvoir la veille sanitaire en santé au travail permettra d'obtenir un état des lieux de la santé de la population salariée afin d'adapter les projets de service et de prioriser les actions de prévention pour in fine consolider le maintien dans l'emploi. Cela passera par la réalisation d'un diagnostic territorial(7).

Pour ce faire, certains services de santé au travail se sont intéressés aux inaptitudes médicales aux postes de travail comme indicateur de la santé des salariés(8).

L'étude de Buewart et al. (2016)(9) réalisé dans le Nord Pas de Calais permet d'identifier un profil type de salarié par le biais de questionnaires mais reste purement descriptive. En l'absence d'analyse des données sur la population suivie parmi laquelle survient les inaptitudes médicales, il est impossible d'évaluer l'incidence des inaptitudes médicales au poste de travail, ni d'analyser les pathologies et les déterminants sociodémographiques et professionnels potentiellement associés aux inaptitudes. De plus, les différentes études réalisées souffrent d'un manque de représentativité par manque de puissance mais également parce que la description des cas d'inaptitude dépend largement du tissu économique du bassin d'emploi étudié.

Dutheil et al. (2014) (10) ainsi que Lesage et al. (2016) (11) se sont intéressés à ce même indicateur qu'est l'inaptitude médicale en analysant directement les données issues du système d'information de deux services de santé au travail distincts et issus de régions différentes. Ces deux études ont permis d'évaluer l'incidence des inaptitudes médicales sur un an, avec des résultats similaires obtenus dans les deux articles. L'analyse sur un an a permis d'observer une incidence moyenne des inaptitudes au poste de travail de 7,8 pour mille salariés tous secteurs confondus. Cependant ces études n'ont pas permis d'identifier des groupes professionnels homogènes, en particulier les plus à risque d'inaptitude en raison d'un faible nombre de cas d'inaptitudes ne permettant que des regroupements de catégories socio professionnelles larges (ex : employés ou ouvriers). 
Ces deux études démontrent néanmoins la capacité de fournir des indicateurs fiables issus des dossiers médicaux en santé en travail(12) et observables dans le temps et l'espace pour in fine l'extrapolation sur l'ensemble du territoire.

L'originalité de notre étude reposera sur la taille de la population suivie, la durée du suivi sur plusieurs années, permettant d'observer une évolution, ainsi que la connaissance de la population de référence.

L'objectif principal de cette étude est d'identifier et décrire des groupes professionnels homogènes ayant une forte incidence des inaptitudes dans un service de santé au travail.

Les objectifs secondaires sont d'observer l'évolution de l'incidence annuelle des inaptitudes médicales au poste de travail dans ce même service de santé au Travail, ainsi que l'identification des pathologies potentiellement responsables dans la survenue de l'inaptitude médicale aux postes de travail. Cette étude s'inscrit également dans les missions de la commission épidémiologique régie par le Comité Régional d'Orientation des Conditions de Travail (CROCT) et mise en place par le groupe de travail 3.13 du Plan Régional Santé Travail (PRST) 2016-2020 Occitanie, dont la mission principale est d'élaborer un outil permettant un recueil harmonisé de données centré sur les inaptitudes. 


\section{Méthodologie :}

Nous avons réalisé une étude épidémiologique prospective sur une cohorte de salariés entre le 1er janvier 2014 et le 31 décembre 2017. L'étude porte sur l'ensemble de la population suivie par les médecins de l'AMETRA, représentant environ 110000 salariés par an répartis dans des secteurs d'activité variés avec une prédominance par le secteur tertiaire.

Critères d'inclusion :

L'ensemble des salariés suivi dans le service de santé au travail entre le 1er janvier 2014 et le 31 décembre 2017 à l'AMETRA.

Critères d'exclusion :

Salariés ayant refusé l'informatisation de son dossier médical de santé au travail.

Recueil des données :

Le recueil et l'analyse statistique suivent la même méthodologie que dans l'étude de Lesage et al. (2016)(11).

L'identification des salariés déclarés inaptes entre 2014 et 2017 a été réalisée par requête informatique dans le système d'information.

Les données sociodémographiques ont été renseignées par tous les médecins du travail, toutes les infirmières diplômées d'état en santé au travail (IDEST) ainsi que les assistantes en santé au travail dans les dossiers médicaux de santé au travail informatisés. Les données cliniques sont enregistrées par les médecins et les IDEST. La prononciation de l'inaptitude ne relève que de la responsabilité des médecins du travail.

L'extraction des données s'est faite en plusieurs étapes à travers différentes requêtes soumises au service informatique du service de santé au travail. Toutes les données extraites étaient enregistrées dans le logiciel métier (logiciel STETHO ${ }^{\circledR}$ ) commun à tous les employés de I'AMETRA (médecins du travail, assistantes médicales, IDEST). Le code d'identification salarié a été extrait dans une première étape. A l'issue du data management (suppression des doublons), il a été supprimé secondairement de la base de données avant analyse permettant d'obtenir une anonymisation complète des données. Les sous-groupes homogènes de métiers pour l'analyse comportaient des effectifs supérieurs à 1000 salariés afin d'assurer l'absence d'identification des cas à posteriori. 
Les différentes données recueillies lors des requêtes sont les données socioprofessionnelles (7 items : l'âge, le sexe, le code de catégorie socioprofessionnelle (PCS) sur plusieurs niveaux, le code de Nomenclature d'activité Française (NAF) sur plusieurs niveaux, la taille de l'entreprise, et l'indice de masse corporel (IMC)).

La deuxième partie du travail porte sur les pathologies pouvant être à l'origine de l'inaptitude.

Lors de la requête ciblant les salariés inaptes médicalement, il a également été recueilli l'année et le mois de l'inaptitude.

Les secteurs d'activité ont été relevés par l'intermédiaire du code NAF (Nomenclature d'Activité Française) utilisé par l'Institut National de la Statistique et des Etudes Economiques (INSEE) en 2008.

La catégorie socioprofessionnelle a été codée en PCS, selon la nomenclature utilisée par I'INSEE (version 2003) en un, deux ou quatre chiffres (plus précis).

Les données sont exportées du logiciel métier sous forme de fichier EXCEL ${ }^{\circledR}$.

La quantité d'adhérents et le nombre de salariés suivis dans un service interentreprises sont en perpétuel mouvement en raison des entrées, sorties, mais globalement identique d'une année sur l'autre. L'analyse des rapports annuels montre que la population instantanée et déclarée est stable à un instant T d'une année sur l'autre. La base de données permettant la description de la population totale de salariés suivis à l'AMETRA par PCS s'est faite en une seule requête à un instant T au cours du mois de Mai 2018 (potentiellement dans un moment ou la déclaration annuelle des effectifs est le plus à jour après de multiples relances faites par le service à destination des employeurs depuis le début d'année). Cette unique base servira au calcul des incidences sur les 4 années de l'analyse. II n'a pas été possible de recueillir la population de salariés suivis spécifique à chaque année car le logiciel ne permettait pas le suivi de la carrière des salariés.

Le taux de remplissage des caractéristiques sociodémographiques pour les salariés au sein du service de santé au travail est excellent. Le renseignement de ces caractéristiques est harmonisé. 
Le niveau 4 des PCS comportent plus de 450 professions. Afin de faciliter la lecture et d'avoir une meilleur visibilité, toutes les PCS INSEE mentionnées dans la base données ont été reprises une par une, et ont été recodées et reclassées de manière à créer des groupes homogènes d'exposition que nous avons nommé PCS AMETRA (19 items).

Le data management et l'analyse descriptive ont été réalisés avec le logiciel $R^{\circledR}$.

Nous avons étudié le lien entre la survenue de l'inaptitude et nos variables d'intérêt en estimant les odds ratios (rapport de cote) avec leurs intervalles de confiance à 95\% à l'aide d'un modèle de régression logistique.

Dans un premiers temps les variables ont été testées en univarié, ensuite toutes les variables significatives à $p<0.20$ (âge, sexe, IMC, PCS) ont été incluses dans un modèle multivarié afin de limiter les facteurs de confusion.

Les tests statistiques ont été réalisés avec le logiciel SASUniversity ${ }^{\circledR}$ (Version 3.6, Copyright 2012-2016, SAS Institute et Inc.,Cary,NC,USA.) 


\section{Résultats :}

La population suivie a été évaluée à 114566 salariés. Parmi cette population, 3614 cas d'inaptitude médicale au poste de travail ont été recueillis sur la période 2014-2017.

L'incidence moyenne annuelle des inaptitudes médicales aux postes de travail est de 7.9 pour mille salariés suivis. Elle est en augmentation constante entre 2014 (7.3\%o) et 2017 (8.3\%o).

Les caractéristiques sociodémographiques des salariés aptes et inaptes sont présentées dans le tableau $n^{\circ} 1$. Les salariés inaptes étaient plus âgés que ceux de la population suivie.

Tableau 1 : Répartition des cas d'inaptitude 2014-2017

\begin{tabular}{|c|c|c|c|c|}
\hline & & $\begin{array}{l}n \text { cas d'inaptitude } \\
4 \text { années }\end{array}$ & n salaries suivis & $\begin{array}{l}\text { Incidence } \\
\text { annuelle } \\
\text { moyenne \%o }\end{array}$ \\
\hline Nombres & & 3614 & 114566 & 7.9 \\
\hline \multirow[t]{5}{*}{ Age } & $<25$ & $101(2.8 \%)$ & $12029(10.5 \%)$ & 2.1 \\
\hline & $25-34$ & 712 (19.7\%) & 31964 (27.9\%) & 5.6 \\
\hline & $35-44$ & 875 (24.2\%) & $28871(25.2 \%)$ & 7.6 \\
\hline & $45-54$ & $943(26.1 \%)$ & $26350(23.0 \%)$ & 8.9 \\
\hline & $>54$ & $983(27.2 \%)$ & $15352(13.4 \%)$ & 16.0 \\
\hline \multirow[t]{2}{*}{ Age moyen par sexe } & Hommes & 45.1 & 39.2 & \\
\hline & femmes & 44.9 & 40.1 & \\
\hline \multirow[t]{2}{*}{ Sexe } & Hommes & 1153 (31.9\%) & $52431(45.8 \%)$ & 5.5 \\
\hline & Femmes & $2461(68.1 \%)$ & $62135(54.2 \%)$ & 9.9 \\
\hline \multirow[t]{7}{*}{ IMC } & $<18,5$ & $76(2.1 \%)$ & $4543(4.0 \%)$ & 4.2 \\
\hline & {$[18,5-25[$} & $868(24.0 \%)$ & $54626(47.7 \%)$ & 4.0 \\
\hline & {$[25-30[$} & $463(12.8 \%)$ & 23908 (20.9\%) & 4.8 \\
\hline & [30-35[ & $184(5.1 \%)$ & $7088(6.2 \%)$ & 6.5 \\
\hline & [35-40[ & $61(1.7 \%)$ & $2013(1.7 \%)$ & 7.6 \\
\hline & $>=40$ & $14(0.4 \%)$ & $636(0.5 \%)$ & 5.5 \\
\hline & $\mathrm{NC}$ & 1948 (53.9\%) & 21752 (19.0\%) & \\
\hline \multirow[t]{4}{*}{ Taille de l'entreprise } & [0 - 9] & $774(21.4 \%)$ & $25892(22.6 \%)$ & 7.2 \\
\hline & [10 - 49] & $1178(32.6 \%)$ & 36661 (32\%) & 8.0 \\
\hline & [50 - 299] & 1297 (35.9\%) & 34943 (30.5\%) & 9.3 \\
\hline & $>299$ & $365(10.1 \%)$ & $17070(14.9 \%)$ & 5.3 \\
\hline \multirow[t]{6}{*}{ PCS 1} & Cadres & $178(4.9 \%)$ & 20637 (18.0\%) & 2.2 \\
\hline & Employés & $1885(52.2 \%)$ & $44026(38.4 \%)$ & 10.7 \\
\hline & Ouvriers & $694(19.2 \%)$ & $14152(12.3 \%)$ & 12.3 \\
\hline & Professions Intermédiaires & $510(14.1 \%)$ & 29664 (25.9\%) & 4.3 \\
\hline & Autres & $27(0.8 \%)$ & $1016(1.0 \%)$ & 6.6 \\
\hline & $\mathrm{NC}$ & $320(8.9 \%)$ & $5071(4.4 \%)$ & \\
\hline \multirow[t]{15}{*}{ NAF 1} & Activ. Ménages : employeurs, produ. & $32(0.9 \%)$ & $121(0.1 \%)$ & 66.1 \\
\hline & Activités de services administratifs et de soutien & $571(15.8 \%)$ & 10619 (9.3\%) & 13.4 \\
\hline & Activités extra-territoriales & $1(0.0 \%)$ & $22(0.0 \%)$ & 11.4 \\
\hline & Activités financières et d'assurance & 109 (3.0\%) & $6389(5.6 \%)$ & 4.3 \\
\hline & Activités immobilières & $74(2.0 \%)$ & $3411(3.0 \%)$ & 5.4 \\
\hline & Activités spécialisées, scientifiques et techniques & $195(5.4 \%)$ & $13840(12.1 \%)$ & 3.5 \\
\hline & Administration publique & $40(1.1 \%)$ & $8132(7.1 \%)$ & 1.2 \\
\hline & Arts, spectacles et activités récréatives & $48(1.3 \%)$ & $2757(2.4 \%)$ & 4.4 \\
\hline & Autres activités de services & $348(9.6 \%)$ & $6445(5.6 \%)$ & 13.5 \\
\hline & $\begin{array}{l}\text { Commerce ; réparation d'automobiles et de } \\
\text { motocycles }\end{array}$ & 741 (20.5\%) & $17319(15.1 \%)$ & 10.7 \\
\hline & Construction & $14(0.4 \%)$ & $760(0.7 \%)$ & 4.6 \\
\hline & Enseignement & $65(1.8 \%)$ & 4150 (3.6\%) & 3.9 \\
\hline & Hébergement et restauration & $406(11.2 \%)$ & 10307 (9.0\%) & 9.8 \\
\hline & Industrie manufacturière & $4(0.1 \%)$ & $284(0.2 \%)$ & 3.5 \\
\hline & Information et communication & $55(1.5 \%)$ & 7569 (6.6\%) & 1.8 \\
\hline
\end{tabular}




\begin{tabular}{l|llll} 
& $\begin{array}{l}\text { Prod. \& distrib. Eau assainisst, gestion déchets \& } \\
\text { dépollution }\end{array}$ & $48(1.3 \%)$ & $1463(1.3 \%)$ & 8.2 \\
& Santé humaine et action sociale & $824(22.8 \%)$ & $19487(17.0 \%)$ & 10.6 \\
& Transports et entreposage & $39(1.1 \%)$ & $1482(1.3 \%)$ & 6.6 \\
& NC & & $9(0.0 \%)$ & 6.5 \\
\hline CSP AMETRA & Accueil / réception de public & $32(0.9 \%)$ & $1233(1.1 \%)$ & 6.7 \\
& Artisans/ouvriers & $62(1.7 \%)$ & $2312(2.0 \%)$ & 10.9 \\
& Autres & $15(0.4 \%)$ & $345(0.3 \%)$ & 11.0 \\
& Coiffure / esthétique & $48(1.3 \%)$ & $1086(0.9 \%)$ & 12.1 \\
& Conduite d'engins / véhicules & $29(0.8 \%)$ & $600(0.5 \%)$ & 8.4 \\
& Emplois d'assainissement et de déchetterie & $42(1.2 \%)$ & $1257(1.1 \%)$ & 3.4 \\
& Emplois pharmaceutiques et de laboratoire & $40(1.1 \%)$ & $2295(2.0 \%)$ & 3.0 \\
& Employés des services sociaux & $22(0.6 \%)$ & $1541(1.3 \%)$ & 9.1 \\
& Enseignement / encadrement & $63(1.7 \%)$ & $5278(4.6 \%)$ & 0.9 \\
& Hôtellerie et Restauration & $326(9.0 \%)$ & $8967(7.8 \%)$ & 8.8 \\
& Ingénieurs & $22(0.6 \%)$ & $6303(5.5 \%)$ & 11.5 \\
& Médicaux et paramédicaux & $325(9.0 \%)$ & $9195(8.0 \%)$ & 2.4 \\
& Métiers de la sécurité/sureté et de la protection & $94(2.6 \%)$ & $2047(1.8 \%)$ & 1.9 \\
& Métiers des arts et spectacles & $10(0.3 \%)$ & $1029(0.9 \%)$ & 18.9 \\
& Métiers du commerce & $769(21.3 \%)$ & $19509(17.0 \%)$ & $12368(10.8 \%)$ \\
& Nettoyage / aide à dom / agents de service & $933(25.8 \%)$ & $27990(24.4 \%)$ & 3.1 \\
& Service de l'administratif et des finances & $352(9.7 \%)$ & $4269(3.7 \%)$ & 2.4 \\
& Techniciens & $41(1.1 \%)$ & $6977(6.1 \%)$ & 13.9 \\
\hline
\end{tabular}


Tableau 2: Evolution des incidences annuelles

\begin{tabular}{|c|c|c|c|c|c|c|c|}
\hline & & $\begin{array}{l}\text { population de } \\
\text { salarie suivi : } \\
\mathrm{n}\end{array}$ & $\begin{array}{l}\text { Incidence des } \\
\text { inaptitudes pour \%o } \\
\text { En 2014 (n) } \\
\end{array}$ & $\begin{array}{l}\text { Incidence des } \\
\text { inaptitudes pour \%o } \\
\text { En } 2015(n) \\
\end{array}$ & $\begin{array}{l}\text { Incidence des } \\
\text { inaptitudes pour \%o } \\
\text { En 2016 (n) } \\
\end{array}$ & $\begin{array}{l}\text { Incidence des } \\
\text { inaptitudes pour \%o } \\
\text { En } 2017(n) \\
\end{array}$ & $\begin{array}{l}\text { Progression } \\
\text { en } \%\end{array}$ \\
\hline Nombre & & 114566 & $7.3(841)$ & $7.4(847)$ & 8.3 (948) & 8.5 (978) & +16 \\
\hline \multirow[t]{5}{*}{ Age } & $<24$ & & 2.2 & 2.3 & 2.4 & 1.5 & \\
\hline & $25-34$ & & 5.4 & 5.1 & 6.1 & 5.7 & \\
\hline & $35-44$ & & 7.4 & 7.6 & 7.5 & 7.9 & \\
\hline & $45-54$ & & 8.7 & 8.7 & 9.0 & 9.7 & +11.5 \\
\hline & $>55$ & & 13.5 & 13.5 & 17.8 & 19.2 & +42 \\
\hline \multirow[t]{2}{*}{ Sexe } & Hommes & & 5.0 & 5.1 & 6.0 & 5.9 & +18 \\
\hline & Femmes & & 9.3 & 9.3 & 10.2 & 10.8 & +16 \\
\hline \multirow[t]{4}{*}{ Taille d'entreprise } & {$[0-9]$} & & 6.9 & 6.3 & 7.7 & 9.0 & \\
\hline & {$[10-49]$} & & 8.0 & 7.7 & 8.4 & 8.1 & \\
\hline & [50 - 299] & & 8.1 & 9.1 & 9.9 & 10.0 & \\
\hline & $>299$ & & 5.2 & 4.9 & 5.4 & 5.8 & \\
\hline \multirow[t]{19}{*}{ NAF } & Activ. Ménages : employeurs, produc. Biens \& serv. (usage propre) & 121 & $66.1(8)$ & $66.1(8)$ & $49.6(6)$ & $82.6(10)$ & +25 \\
\hline & Activités de services --administratifs et de soutien & 10619 & $14.6(155)$ & $11.8(125)$ & $13.0(138)$ & 14.4 (153) & \\
\hline & Activités extra-territoriales & 22 & 0.0 & $45.5(1)$ & 0.0 & 0.0 & \\
\hline & Activités financières et d'assurance & 6389 & $3.6(23)$ & $3.4(22)$ & $5.6(36)$ & $4.4(28)$ & \\
\hline & Activités immobilières & 3411 & $5.0(17)$ & $3.2(11)$ & $6.7(23)$ & $6.7(23)$ & \\
\hline & Activités spécialisées, scientifiques et techniques & 13840 & $3.7(51)$ & $4.0(56)$ & $3.0(41)$ & $3.4(47)$ & \\
\hline & Administration publique & 8132 & $1.6(13)$ & $1.1(9)$ & $1.1(9)$ & $1.1(9)$ & \\
\hline & Arts, spectacles et activités récréatives & 2757 & 4.7 (13) & $4.7(13)$ & $3.3(9)$ & $4.7(13)$ & \\
\hline & Autres activités de services & 6445 & $12.1(78)$ & $10.6(68)$ & $16.9(109)$ & $14.4(93)$ & +19 \\
\hline & Commerce ; réparation d'automobiles et de motocycles & 17319 & 9.4 (163) & $10.7(185)$ & $11.0(191)$ & $11.7(202)$ & +24 \\
\hline & Construction & 760 & $1.3(1)$ & $3.9(3)$ & $6.6(5)$ & $6.6(5)$ & \\
\hline & Enseignement & 4150 & $3.4(14)$ & $4.1(17)$ & $3.1(13)$ & $5.1(21)$ & \\
\hline & Hébergement et restauration & 10307 & $7.5(77)$ & $9.6(99)$ & $11.4(117)$ & $11.0(113)$ & +46 \\
\hline & Industrie manufacturière & 284 & $10.6(3)$ & $3.5(1)$ & 0.0 & 0.0 & \\
\hline & Information et communication & 7569 & $1.3(10)$ & 1.8 (14) & $2.0(15)$ & $2.1(16)$ & \\
\hline & Prod. \& distrib. Eau assainisst, gestion déchets \& dépollution & 1463 & $9.6(14)$ & $6.2(9)$ & $8.9(13)$ & $8.2(12)$ & \\
\hline & Santé humaine et action sociale & 19487 & $9.8(191)$ & $10.3(200)$ & $10.8(210)$ & $11.4(223)$ & +16 \\
\hline & Transports et entreposage & 1482 & $6.7(10)$ & $4.0(6)$ & $8.8(13)$ & $6.7(10)$ & \\
\hline & (vide) & 44 & - & - & - & - & \\
\hline \multirow[t]{10}{*}{ CSP AMETRA } & Accueil / réception de public & 1233 & $7.3(9)$ & $5.7(7)$ & $7.3(9)$ & $5.7(7)$ & \\
\hline & Artisans/Ouvriers & 2312 & $4.8(11)$ & $7.4(17)$ & $8.2(19)$ & $6.5(15)$ & \\
\hline & Autres & 345 & $11.6(4)$ & $8.7(3)$ & $14.5(5)$ & $8.7(3)$ & \\
\hline & Coiffure / esthétique & 1086 & $5.5(6)$ & $6.4(7)$ & $15.7(17)$ & $16.6(18)$ & +200 \\
\hline & Conduite d'engins / véhicules & 600 & $15.0(9)$ & $11.7(7)$ & $15.0(9)$ & $6.7(4)$ & \\
\hline & Emplois d'assainissement et de déchetterie & 1257 & $9.5(12)$ & $6.4(8)$ & $8.8(11)$ & $8.8(11)$ & \\
\hline & Emplois pharmaceutiques et de -laboratoire & 2295 & 3.9 (9) & $5.7(13)$ & $3.5(8)$ & $4.4(10)$ & \\
\hline & Employés des services sociaux & 1541 & $3.2(5)$ & $3.2(5)$ & $2.6(4)$ & $5.2(8)$ & \\
\hline & Enseignement / Encadrement & 5278 & $2.5(13)$ & $3.0(16)$ & $4.0(21)$ & $2.5(13)$ & \\
\hline & Hôtellerie et Restauration & 8967 & $6.1(55)$ & $9.3(83)$ & $11.0(99)$ & $9.9(89)$ & +62 \\
\hline
\end{tabular}


Ingénieurs

Médicaux et paramédicaux

6303

$0.5(3)$

1.1 (7)

$6.3(58)$

$7.3(15)$

$2.9(3)$

$2.9(3)$

$8.2(75)$

12.7 (26)

2.9 (3)

Métiers des arts et spectacles

1029

$8.9(173)$

$9.6(187)$

Nettoyage / aide à dom / agents de service

1950

$18.3(226)$

$16.0(198)$

Service de l'administratif et des finances

$2.9(80)$

3.1 (86)

$\begin{array}{ll}27990 & 2.9(80) \\ 4269 & 2.3(10) \\ 6977 & (140)\end{array}$

$1.0(6)$

10.2 (94)

$10.7(22)$

$1.9(2)$

9.8 (191)

$20.1(248)$

$3.1(88)$

$1.0(6)$

10.7 (98)

$15.1(31)$

$1.9(2)$

+69
+106

(vide)

6977

3.5 (98)

$3.3(14)$

$+16$ 
Tableau 3 : Analyse multivariée des facteurs associés à l'inaptitude médicale

\begin{tabular}{|c|c|c|c|}
\hline & & OR [IC95\%] & $\mathbf{p}$ \\
\hline \multirow[t]{2}{*}{ Sexe } & Hommes & 1 & \\
\hline & Femmes & $1.68[1.55 ; 1.82]$ & $<0.0001$ \\
\hline \multirow[t]{3}{*}{ Age } & $<40$ ans & 1 & \\
\hline & {$[40-49]$ ans } & $1.60[1.46 ; 1.75]$ & $<0.0001$ \\
\hline & $>49$ ans & $2.10[1.93 ; 2.29]$ & $<0.0001$ \\
\hline \multirow[t]{2}{*}{ IMC } & $<25$ & 1 & \\
\hline & $>=25$ & $1.27[1.14 ; 1.40]$ & $<0.0001$ \\
\hline \multirow{4}{*}{ CSP 1} & Cadres & 1 & \\
\hline & Employés & $4.58[3.91 ; 5.35]$ & $<0.0001$ \\
\hline & Ouvriers & $5.61[4.74 ; 6.63]$ & $<0.0001$ \\
\hline & Professions intermédiaires & $1.97[1.66 ; 2.34]$ & $<0.0001$ \\
\hline \multirow[t]{12}{*}{ NAF 1} & Activités financières et d'assurance & 1 & \\
\hline & Commerce ; réparation d'automobiles et de motocycles & $3.42[2.54 ; 4.60]$ & $<0.0001$ \\
\hline & Activités de services administratifs et de soutien & $3.15[2.31 ; 4.28]$ & $<0.0001$ \\
\hline & Activités spécialisées, scientifiques et techniques & $0.77[0.53 ; 1.10]$ & 0.15 \\
\hline & Autres activités de services & $3.04[2.21 ; 4.20]$ & $<0.0001$ \\
\hline & Activ. Ménages : employeurs, produc. Biens \& serv. (usage propre) & $12.88[6.94 ; 23.90]$ & $<0.0001$ \\
\hline & Construction & $1.45[0.68 ; 3.07]$ & 0.33 \\
\hline & Enseignement & $1.04[0.67 ; 1.61]$ & 0.85 \\
\hline & Hébergement et restauration & $3.52[2.56 ; 4.83]$ & $<0.0001$ \\
\hline & Prod. \& distrib. Eau assainisst, gestion déchets \& dépollution & $2.71[1.65 ; 4.45]$ & $<0.0001$ \\
\hline & Santé humaine et action sociale & $2.41[1.79 ; 3.24]$ & $<0.0001$ \\
\hline & Autres NAF & $0.78[0.56 ; 1.09]$ & 0.15 \\
\hline \multirow[t]{13}{*}{ CSP AMETRA } & Service de l'administratif et des finances & 1 & \\
\hline & Accueil / réception de public & $1.38[0.73 ; 2.62]$ & 0.32 \\
\hline & coiffure / esthétique & $4.91[3.22 ; 7.50]$ & $<0.0001$ \\
\hline & Conduite d'engins / véhicules & $6.65[4.03 ; 10.96]$ & $<0.0001$ \\
\hline & Emplois d'assainissement et de déchetterie & $4.44[2.78 ; 7.10]$ & $<0.0001$ \\
\hline & Emplois pharmaceutiques et de laboratoire & $1.70[1.06 ; 2.64]$ & 0.03 \\
\hline & Enseignement / Encadrement & $1.12[0.76 ; 1.66]$ & 0.56 \\
\hline & Hôtellerie et Restauration & $4.96[3.96 ; 6.20]$ & $<0.0001$ \\
\hline & Médicaux et paramédicaux & $3.11[2.49 ; 3.87]$ & $<0.0001$ \\
\hline & Métiers de la sécurité/sureté et de la protection & $6.19[4.33 ; 8.66]$ & $<0.0001$ \\
\hline & Métiers du commerce & $4.18[3.47 ; 5.05]$ & $<0.0001$ \\
\hline & Nettoyage / aide à dom / agents de service & $5.17[4.29 ; 6.23]$ & $<0.0001$ \\
\hline & Autres CSP AMETRA & $2.71[2.22 ; 3.31]$ & $<0.0001$ \\
\hline
\end{tabular}

Après ajustement sur le sexe, l'âge et l'IMC, les salariés les plus à risque d'inaptitude sont les femmes (OR 1.68, IC 95\% [1.55; 1.82]), les salariés de plus de 50 ans (OR 2.10, IC 95\% [1.93 ; 2.29]) et les salariés en surpoids (OR 1.27, IC 95\% [1.14; 1.40]). La taille des entreprises ne présentait pas de lien avec le risque d'inaptitude.

L'analyse par secteur d'activité (tableau 2), fait apparaitre une progression importante associée à des incidences au-dessus de celle de la population générale dans différents secteurs comme les activités de ménages en tant qu'employeurs ; les autres activités de services ; le commerce, la réparation d'automobiles et de motocycles; la santé humaine et l'action sociale ; l’hébergement 
et la restauration. Ces secteurs ont été individuellement comparés après ajustement au secteur des activités financières et d'assurances en raison des faibles incidences qu'il présente. Les risques les plus importants étaient observés dans le secteur des activités des ménages en tant qu'employeurs (OR 12.88 IC 95\% [6.94; 23.90]) malgré le petit effectif de 121 personnes suivies, I'hébergement et la restauration (OR 3.52 IC 95\% [2.56; 4.83]) et celui du commerce (OR 3.42 IC $95 \%[2.54 ; 4.60])$.

L'incidence la plus faible était observée chez les cadres qui ont été choisis comme population de référence. Etre ouvrier (OR 5.61 IC 95\% [4.74; 6.63]) et employé (OR 4.58 IC 95\% [3.91; 5.35]) était sensiblement lié à la survenue de l'inaptitude comparé aux cadres.

En examinant les professions concernées dans la classe " employés », nous avons constaté qu'il s'agissait d'employés de commerce (vendeur, caissier, employé de libre-service) et de personnels des services directs aux particuliers (femmes de ménage, aides à domicile...). Ces professions regroupent $65 \%$ des inaptitudes sur la totalité des « employés » qui eux représentent $38.5 \%$ de la population suivie.

Si l'on examine les inaptitudes dans la classe « ouvriers », il s'agissait dans $70 \%$ des cas d'ouvriers qualifiés et non qualifiés de type artisanal. Les ouvriers qualifiés étant essentiellement représentés par les métiers de la restauration (cuisiniers, commis...) et les non qualifiés par les agents d'entretien, aides à domicile. Les $30 \%$ restants se partageaient entre les chauffeurs, les ouvriers non qualifiés de type industriel et les ouvriers qualifiés de la manutention.

Le regroupement des professions par groupe homogène d'exposition en PCS AMETRA, a permis d'identifier les plus grandes incidences pour le groupe « nettoyage, aides à domicile, agents de service » avec une incidence annuelle moyenne de 18.9 inaptitudes pour mille salariés suivis. En effet, ce groupe recense $25.8 \%$ des cas d'inaptitude alors qu'il représente $10.8 \%$ de la population suivie. Après ajustement, ce groupe présentait un des plus hauts rapports de cote (OR 5.17 IC $95 \%$ [4.29; 6.23]) associé à la survenue de l'inaptitude comparé aux professions des « services de l'administratifs et des finances ". La plus forte évolution d'incidence était observée chez les coiffeurs/esthétiques (+200\%).

Le groupe professionnel « nettoyage, aides à domicile, agent de service » est constitué de $81 \%$ de femmes. L'âge moyen est de 47.4 ans. Cette population est essentiellement répartie dans le 
secteur de la santé humaine et action sociale (36\%), dans les activités de services administratifs et de soutien (33\%), ainsi que dans les autres activités de service (20\%).

La phase de data management n'a retenu que 1800 cas d'inaptitudes toutes professions confondues pour l'analyse des pathologies. Parmi les pathologies renseignées dans ces dossiers, les troubles de l'appareil locomoteur (46.1\%), essentiellement représentées par des atteintes du rachis, et les atteintes psychiatriques (43.4\%), notamment les dépressions, prédominent.

Dans le groupe professionnel « nettoyage, aides à domicile, agent de service ", une diminution du taux d'atteintes ostéo-articulaires sur les trois dernières années est constatée (de $30.6 \%$ en 2015 à 25.1\% en 2017) alors que la tendance est inversée pour les troubles mentaux et du comportement (passant de $26.2 \%$ en 2015 à 34.4\% en 2017) (tableau 4).

Tableau 4 : évolution des pathologies déclarées dans le groupe nettoyage sur 4 ans

\begin{tabular}{|c|c|c|c|c|c|c|c|c|}
\hline \multirow[b]{2}{*}{$\mathrm{n}$ inaptitudes } & \multicolumn{2}{|c|}{2014} & \multicolumn{2}{|c|}{2015} & \multicolumn{2}{|c|}{2016} & \multicolumn{2}{|c|}{2017} \\
\hline & $\begin{array}{c}\text { Nettoyages } \\
196\end{array}$ & $\begin{array}{c}\text { Autres } \\
\text { métiers } \\
459\end{array}$ & $\begin{array}{c}\text { Nettoyages } \\
183\end{array}$ & $\begin{array}{c}\text { Autres } \\
\text { métiers } \\
535\end{array}$ & $\begin{array}{c}\text { Nettoyages } \\
226\end{array}$ & $\begin{array}{c}\text { Autres } \\
\text { métiers } \\
591\end{array}$ & $\begin{array}{c}\text { Nettoyages } \\
247\end{array}$ & $\begin{array}{c}\text { Autres } \\
\text { métiers } \\
621\end{array}$ \\
\hline $\begin{array}{l}\text { Troubles osteo- } \\
\text { articulaires }\end{array}$ & 34 (17.3\%) & 74 (16.1\%) & $56(30.6 \%)$ & 164 (30.6\%) & 65 (28.7\%) & $166(28.1 \%)$ & $62(25.1 \%)$ & 192 (30.9\%) \\
\hline $\begin{array}{l}\text { Troubles } \\
\text { psychiatriques }\end{array}$ & 37 (18.9\%) & 87 (18.9\%) & $48(26.2 \%)$ & 154 (28.8\%) & 83 (36.7\%) & 188 (31.8\%) & 85 (34.4\%) & $200(32.2 \%)$ \\
\hline Divers & $8(4.1 \%)$ & 16 (3.5\%) & $9(4.9 \%)$ & 35 (6.5\%) & 19 (8.5\%) & 44 (7.5\%) & 25 (10.1\%) & 47 (7.5\%) \\
\hline
\end{tabular}

Après regroupement des autres atteintes (cardiaques, pulmonaires, oncologiques, neurologiques, ...), nous notons une augmentation du nombre de ces pathologies déclarées dans notre échantillon contre une stabilité des déclarations annuelles pour le reste des professions.

Le groupe professionnel " nettoyage, aides à domicile, agent de service " concentre à lui-seul 33.2\% des troubles dépressifs déclarés, $24.6 \%$ des atteintes du rachis déclarées, $26 \%$ des TMS MS, $29.4 \%$ des troubles bipolaires déclarés, $26.2 \%$ des cancers déclarés, $48.1 \%$ des pathologies cardiaques et $27 \%$ des atteintes pulmonaires. 
Tableau 5 : Fréquence d'apparition des pathologies dans la catégorie nettoyage

\begin{tabular}{lll}
\hline Pathologies & Nettoyages : $\mathbf{n}$ & $\begin{array}{l}\text { Toutes } \\
\text { confondues }: \mathbf{n}\end{array}$ \\
\hline TMS MS & 48 & 184 \\
TMS MI & 10 & 58 \\
RACHIS & 184 & 748 \\
SPA & 2 & 7 \\
PR & 6 & 28 \\
\hline Trouble anxio-dépressif & 248 & 966 \\
Trouble bipolaire & 5 & 17 \\
Schizophrénie & 2 & 5 \\
\hline Cardiaque & 13 & 17 \\
Pneumo & 17 & 63 \\
Cancers & 11 & 27 \\
\hline
\end{tabular}

En dépit de la forte incidence dans cette population, le nombre de cas d'inaptitude augmente moins vite dans cette famille (+16\%) que pour les autres professions confondus $(+35 \%)$. 


\section{Discussion :}

L'analyse de la population suivie dans ce service de santé au travail du sud de la France et des inaptitudes recueillies sur 4 ans, permet d'identifier les femmes de plus de 45 ans et en surpoids comme étant plus à risque d'inaptitude. Ce profil est retrouvé dans les différentes études $(13,14)$. Une étude réalisée dans le Var en 2016(15) permet d'observer l'évolution du profil avant et après les années 2000, passant d'un homme de plus de 50 ans à une femme de 45 ans en moyenne(13).

L'avis d'inaptitude prononcé par le médecin du travail engendre un risque très important de perte d'emploi et d'exclusion sociale. L'anticipation de cet évènement passe par une meilleure connaissance de la population à risque. Plus de $90 \%$ des cas d'inaptitudes aboutissent à un licenciement professionnel $(16,17)$. Le risque d'inaptitude lié à l'âge avancé rendent encore plus difficile les missions de maintien dans l'emploi, en raison des difficultés de reclassement et de l'augmentation de la fréquence et de la gravité des pathologies avec I'âge(18). Ces deux facteurs font partie intégrante des préoccupations du PRST Occitanie.

L'analyse de l'incidence des inaptitudes, de ses paramètres associés(19) et son évolution dans le temps apparait comme étant un indicateur de santé pertinent. Cet indicateur de santé est commun à tous les services de santé au travail et permettrait de cibler des populations à risque élevé d'inaptitude, de repérer l'émergence d'une population à risque (ex coiffure) et ainsi d'agir en amont par des actions de prévention ciblées. II permettrait également d'observer les effets positifs ou négatifs dans le temps d'une action de prévention encadrée par les Contrats Pluriannuels d'Objectifs et de Moyens (CPOMs) dans les services de santé au travail.

Nous constatons dans notre étude, qu'un tiers de la population de référence est en surpoids. Malgré le nombre important de données manquantes, un lien significatif existe entre I'IMC et le risque d'inaptitude. Nous savons qu'un IMC élevé est pourvoyeur de maladies chroniques ou pathologies(20) pouvant être en lien avec le travail(21). II semblerait judicieux de s'intéresser à ce facteur dans une autre étude. Les données recueillies pourraient par ailleurs être utiles à participer à la veille sanitaire des problèmes de santé publique et ainsi répondre à la recommandation $n^{\circ} 5$ du rapport Lecocq (Annexe 2). 
La proportion d'inaptes la plus faible se trouve être dans les entreprises de 299 salariés et plus. Cela est probablement lié à une plus grande capacité d'aménagement de poste et de reclassement réalisable avant la mise en inaptitude par le médecin. En effet, ces entreprises disposent régulièrement de préventeurs ou responsables HSE (sécurité, hygiène, environnement) facilitant la collaboration avec les médecins du travail.

Nous enregistrons de fortes progressions dans des secteurs d'activité avec des incidences plus faibles qui laisse penser qu'une action primaire de prévention serait nécessaire avant d'atteindre un score similaires (7.9\%o salariés) à celui de la population générale. Ces secteurs sont les secteurs de l'enseignement, de la construction et des activités immobilières. Mais I'hétérogénéité des catégories socioprofessionnelles dans chaque secteur rend nécessaire une analyse approfondie. Le département des affaires régionales, de l'économie et de la santé (DARES) de Genève, Suisse semble montrer que les conditions de travail sont plus étroitement dépendantes du métier exercé par le salarié, classé par famille professionnelle, que le secteur d'activité de l'établissement où il exerce, et que les risques professionnels sont souvent attachés à des métiers spécifiques(22).

Les ouvriers et les employés sont surreprésentés comparés à la place qu'ils occupent dans la population de référence inversement aux professions intermédiaires et aux cadres. Cela permet d'appuyer l'hypothèse qu'il existerait un lien entre le niveau d'études et le risque d'inaptitude(23).

L'analyse médicale des cas d'inaptitude fait apparaitre dans un premier temps une différence d'âge et de la répartition par sexe entre les dossiers avec des pathologies renseignées et ceux sans pathologies renseignées. Cette différence est probablement liée à l'un des plus gros biais de sélection de notre étude, celui de l'enquêteur. En effet la répartition des secteurs d'activités par médecin se faisant par secteur géographique, il est impossible d'obtenir une homogénéité du type de salarié suivi par médecin. De plus, la maitrise et l'utilisation du logiciel métier d'un médecin à l'autre est totalement inégale créant un manque d'harmonisation dans le recueil de données cliniques. Effectivement, le data management n'a permis de retenir que 1800 (soit 50\%) cas d'inaptitudes pour l'analyse des pathologies. Le constat est que probablement plus de la moitié (biais enquêteur) des médecins ne renseignent aucune donnée médicale statistiquement analysable dans le DSMT. La réalisation d'un bon diagnostic territorial et la garantie de mener à bien sa mission de veille sanitaire par 
les services de santé au travail, passera par une sensibilisation des médecins du travail au recueil des données.

Néanmoins, les résultats obtenus affichent une prédominance des pathologies ostéoarticulaires et psychiatriques comparable aux données de la littérature et aux enquêtes réalisées sur les inaptitudes(10,11,24). II reste pourtant encore difficile de dire si la décompensation ou le développement d'un trouble psychiatrique est imputable ou non à la profession ou un environnement de travail. C'est pourquoi les pathologies psychiatriques ne font toujours pas partie du tableau des maladies professionnelles et sont passés en commission régionale de reconnaissance des maladies professionnelles (CRRMP) avant d'être reconnue en tant que telle(25), malgré la proportion importante qu'ils occupent dans les dossiers comparés au début des années 2000.

Il semble exister un lien entre la décompensation de certaines pathologies(26) et notamment des pathologies psychiatrique et la saisonnalité $(27,28)$. Un projet futur sera de rechercher un tel lien pour le risque d'inaptitude(29).

Il est intéressant d'observer des populations telles que celle des nettoyages, aides à domicile et agents de service en raison de l'importance de l'incidence des inaptitudes dans cet échantillon. Cette population est initialement connue comme à risque élevé de TMS(30). II existe, sur les dernières années, une tendance inversée de la fréquence d'apparition des pathologies comparé au reste de la population inapte avec une augmentation du nombre de pathologies psychiatriques et une diminution du nombre de pathologies ostéo-articulaires. Dans le service interentreprise qui a fait l'objet de cette étude, à la suite du CPOM (20132018), un groupe de travail « aides à domicile " s'est mis en place depuis 2014. L'analyse des incidences démontre une stabilité des incidences depuis 2016. Cependant, ce groupe de travail axe ses actions en ciblant les TMS et les risques biologiques. L'observation des pathologies semble indiquer la nécessité de recentrer les actions dans ce groupe vers les risques psychosociaux. En plus de leur exposition importante aux facteurs de risques de TMS, cette profession recrute plus facilement une population fragile concentrant les problèmes de santé, ce qui peut expliquer les fortes incidences observées et permettre d'être plus vigilant lors du suivi des salariés. Les études retrouvent également un taux de dépression plus élevé chez les pourvoyeurs de soins aux personnes âgés ou avec des troubles cognitifs en cas de facteurs de stress personnels ou d'instabilité sociale. C'est le cas des aides à domiciles qui 
comme nous l'avons retrouvé ont déjà des comorbidités médicales. Les problèmes de santé physique surviennent plus facilement chez des personnes psychologiquement en détresse(31,32).

Notre étude permet ainsi d'obtenir un état des lieux de la santé globale d'une sous population par l'observation de l'évolution du nombre de pathologies autres que professionnelles déclarées.

Une des limites de notre étude reste le fait qu'un salarié déclaré inapte, sort des effectifs du service et est perdu de vue. II reste donc difficile d'analyse l'effet temps et l'impact d'une mise en inaptitude médicale sur le devenir des salariés en terme de changement de PCS, d'évolution des pathologies et autres. Le seul moyen d'étudier la carrière d'un salarié en détail serait l'élaboration d'un curriculum laboris de chaque salarié mis en inaptitude par le médecin du travail.

Une autre limite est que ce service de santé au travail assure également le suivi d'agents de la fonction publique ( $2 \%$ de la population totale étudiée). Hors, un médecin du travail ne peut prononcer une inaptitude médicale pour cette catégorie de travailleurs, le système étant différent (comité médical). De plus 10\% des salariés suivis sont en contrat à durée déterminée (CDD). Il est difficile de mesurer la survenue d'une inaptitude dans cet échantillon car la plupart des salariés quittent d'eux-mêmes leur environnement de travail en cas de difficultés. Il en est de même pour les salariés en période d'essai. Tous ces facteurs contribuent à une sous-évaluation de l'incidence des inaptitudes.

Cette étude nous permet de démontrer le panel de tout ce qui est réalisable avec les informations issues du DSMT et une grande puissance. Le logiciel métier ne nous permet pas encore de renseigner un lien statistiquement analysable entre l'inaptitude et les conditions de travail ou une pathologie.

La surveillance est une étape fondamentale pour mesurer l'évolution de l'état de santé d'une population. Avec l'augmentation croissante des cas d'inaptitude et le vieillissement de la population, la surveillance jouera un rôle essentiel dans l'amélioration des mesures de prévention et la lutte contre la désinsertion professionnelle. Les services de santé au travail regorgent d'informations exploitables pouvant contribuer à cette surveillance en identifiant 
des indicateurs de santé fiable. Il semble donc intéressant de développer un outil commun de partage des données et de promouvoir l'interopérabilité des différents services.

C'est dans l'intérêt d'améliorer la qualité des données des DSMT et de mieux adapter les objectifs de projet de service, que la commission épidémiologique Occitanie (régie par la DIRECCTE et composée de représentant de la CARSAT, Santé Publique France, Universitaires, ORD, ARACT et les Services de Santé au Travail) a été créée. L'objectif principal étant de répondre à la problématique d'associer une pathologie à l'inaptitude et ainsi développer un outil de veille sanitaire en direct, permettant une harmonisation dans le recueil et le partage de cette information pour in fine améliorer la vigilance. Actuellement le seul moyen d'obtenir ce genre d'information, passe par la réalisation d'enquête réalisée à l'échelle locale ou régionale telle l' "enquête inaptitude ". Des systèmes de recueils de données en direct à l'échelle nationale ont été développés dans d'autres pays et ont prouvé leur efficacité(33).

Les facteurs psychosociaux et la manutention ne sont pas repris dans la liste des situations de travail impliquant légalement une surveillance médicale renforcée, alors qu'ils sont très souvent en cause dans les licenciements pour inaptitudes et certainement aussi dans un grand nombre de cas de morbidité.

La prescription des périodicités du suivi des salariés en santé au travail est réglementée et se découpe en 3 catégories: SIR (suivi individuel renforcé), SIA (suivi individuel adapté) et SI (suivi individuel simple), modulable par les médecins du travail selon l'état de santé des salariés. Certains médecins souhaiteraient une aide quant à l'adaptation du suivi des salariés. Notre étude nous aura permis de de démontrer que l'analyse des données sociodémographique en grande quantité, ajustées sur les variables de confusions permet d'obtenir des résultats statistique hautement significatif quant au risque d'inaptitude. Ce type de résultat pourrait aider à adapter la périodicité dans le suivi des salariés en fonction des professions et de l'incidence des inaptitudes dans leur catégorie. 


\section{Conclusion :}

Les services de santé au travail regorgent de données non exploitées. Notre étude permet de démontrer les champs explorables et les limites à travers les données extraites de logiciels métiers.

L'analyse des dossiers médicaux en santé au travail permet d'affiner l'état de santé de la population active et de mettre en évidence les problématiques de maintien à l'emploi. Ainsi 3614 cas d'inaptitude au poste de travail ont été analysés sur 4 années, entre 2014 et 2017.

Des secteurs professionnels et des métiers ont été identifiés comme étant plus à risque de désinsertion professionnelle. Ce constat permettra d'orienter la mise en place des plans de prévention et le projet du service étudié.

Cette étude montre également la faisabilité de la veille sanitaire en santé au travail au niveau local. La mise en commun des données au niveau régional permettrait d'élaborer une cartographie de l'état de santé des travailleurs afin d'adapter les politiques de santé.

Cependant une harmonisation des pratiques pour le recueil ou la promotion d'outils plus efficace est indispensable afin d'obtenir des informations qualitativement exploitables. La force du système de santé au travail français est que toute personne active dispose d'un dossier médical en santé au travail. 


\section{Bibliographie}

1. Tuppin P, Rudant J, Constantinou P, Gastaldi-Ménager C, Rachas A, de Roquefeuil L, et al. Value of a national administrative database to guide public decisions: From the système national d'information interrégimes de l'Assurance Maladie (SNIIRAM) to the système national des données de santé (SNDS) in France. Rev Epidemiol Sante Publique. oct 2017;65 Suppl 4:S14967.

2. Chan Chee C, Paget L-M. [The psychiatric hospital discharge database (RIM-P): An essential tool for the surveillance of hospitalization after a suicide attempt]. Rev Epidemiol Sante Publique. sept 2017;65(5):349-59.

3. Molinié A-F, Leroyer A. Suivre les évolutions du travail et de la santé : EVREST, un dispositif commun pour des usages diversifiés. Perspect Interdiscip Sur Trav Santé [Internet]. 1 nov 2011 [cité 28 sept 2018];(13-2). Disponible sur: http://journals.openedition.org/pistes/1852

4. Bonneterre V, Faisandier L, Bicout D, Bernardet C, Piollat J, Ameille J, et al. Programmed health surveillance and detection of emerging diseases in occupational health: contribution of the French national occupational disease surveillance and prevention network (RNV3P). Occup Environ Med. mars 2010;67(3):178-86.

5. recommendation dossier medical de santé au travail - Disponible sur: https://www.google.fr/search?q=recommendation+dossier+medical+de+sant\%C3\%A9+au+tra vail\&rlz=1C1CHBF_frFR813FR813\&oq=recommendation+dossier+medical+de+sant\%C3\%A9+au +travail\&aqs=chrome..69i57j0.11899j1j7\&sourceid=chrome \&ie=UTF-8

6. THEME 8 : La santé au travail / Atlas Santé / Accueil CREAI-ORS LR - Tél. 0467692503 - CREAI LANGUEDOC ROUSSILLON [Internet] Disponible sur: http://www.creaiorsIr.fr/AtlasSante/THEME-8-La-sante-au-travail

7. Chapon P-M, Pihet C, Jahan F, Michel B, Riobe A-L, Merjagnan-Vilcocq C, et al. [The DATEL (diagnosis for territorial action environment and longevity), a territorial diagnosis for a future with our elders]. Geriatr Psychol Neuropsychiatr Vieil. juin 2012;10(2):123-7.

8. Jegaden $D$, Nousbaum $M$, Tepaut B. Un indicateur pertinent de santé au travail : le suivi annuel de critères associésaux inaptitudes définitives. Arch Mal Prof Environ. 1 mai 2006;67(2):367.

9. Buewaert $V$, Surquin $M$, Leroyer A. Suivi d'indicateurs régionaux sur les inaptitudes médicales en Nord Pas-de-Calais. Arch Mal Prof Environ. 1 juin 2016;77(3):375-6.

10. Dutheil F, Naughton G, Sindyga P, Lesage F-X. III Health-Related Job Loss: A One-Year Follow-Up of 54,026 Employees. J Occup Environ Med. sept 2016;58(9):918-23.

11. Lesage F-X, Dutheil F, Godderis L, Divies A, Choron G. Incidence of ill-health related job loss and related social and occupational factors. The « unfit for the job » study: a one-year follow-up study of 51,132 workers. PeerJ. 2018;6:e5073.

12. Lesage F-X, Divies A, Choron G. Extraction et analyse des données issues des dossiers de santé au travail : principe et perspectives. Arch Mal Prof Environ. 1 juin 2016;77(3):439.

13. Maysonnave $\mathrm{C}$, Tagorti $\mathrm{S}$, Bessieres $\mathrm{P}$, Fernet $\mathrm{F}$, Dalm C. Caractéristiques des salariés déclarés inaptes définitifs en Aquitaine en 2003 : portrait type et perspectives en matière d'aide au maintien dans l'emploi. Arch Mal Prof Environ. 1 mai 2004;65(2):227. 
14. Buchet C, Col A, de Labrusse B, Rigaut H, Masse A-M, Faivre-Dupaigre M. Devenir des salariés licenciés suite à une inaptitude au poste de travail en Vaucluse de 2002 à 2004. Arch Mal Prof Environ. 1 mai 2010;71(2):108-16.

15. Baju-Deniaud A, Viola A, Charpentier P, Dussarat $M$. Inaptitudes définitives dans le var : quelle évolution en 20 ans ? Arch Mal Prof Environ. 1 mai 2018;79(3):455.

16. Rondi A, Fantoni-Quinton S. Devenir des salariés après une inaptitude médicale. Arch Mal Prof Environ. 1 oct 2017;78(5):412-20.

17. Trajectoires Inaptitudes - Direccte Bretagne [Internet]. [cité 5 sept 2018]. Disponible sur: http://bretagne.direccte.gouv.fr/trajectoires-inaptitudes

18. Leijten FRM, van den Heuvel SG, Ybema JF, van der Beek AJ, Robroek SJW, Burdorf A. The influence of chronic health problems on work ability and productivity at work: a longitudinal study among older employees. Scand J Work Environ Health. sept 2014;40(5):473-82.

19. Haahr JPL, Frost P, Andersen JH. Predictors of health related job loss: a two-year follow-up study in a general working population. J Occup Rehabil. déc 2007;17(4):581-92.

20. Bonde JPE, Viikari-Juntura E. The obesity epidemic in the occupational health context. Scand J Work Environ Health. 1 mai 2013;39(3):217-9.

21. Arena VC, Padiyar KR, Burton WN, Schwerha JJ. The impact of body mass index on short-term disability in the workplace. J Occup Environ Med. nov 2006;48(11):1118-24.

22. Amira, D Ast. Des risques professionnels contrastés selon les métiers. Dares-Analyses; №39, Mai 2014. Disponible à partir de l'URL: http://dares.travail-emploi.gouv.fr - Recherche Google [Internet]. Disponible sur:

https://www.google.com/search?q=Amira\%2C+D+Ast.+Des+risques+professionnels+contrast\% C3\%A9s+selon+les+m\%C3\%A9tiers.+Dares-

Analyses\%3B+N\%C2\%B0039\%2C+Mai+2014.+Disponible+\%C3\%A0+partir+de+|\%E2\%80\%99UR L\%3A+http\%3A\%2F\%2Fdares.travail-emploi.gouv.fr\&ie=utf-8\&oe=utf-8\&client=firefox-b-ab

23. Schuring $\mathrm{M}$, Robroek SJW, Otten FWJ, Arts $\mathrm{CH}$, Burdorf A. The effect of ill health and socioeconomic status on labor force exit and re-employment: a prospective study with ten years follow-up in the Netherlands. Scand J Work Environ Health. 1 mars 2013;39(2):134-43.

24. MANET C. L'inaptitude médicale au poste de travail: enquête épidémiologique descriptive dans trois services de santé au travail de Meurthe et Moselle en 2007-158p. Th.D.: Médecine du travail: Nancy: 2008

25. INRS, 2012, Les maladies professionnelles - Guide d'accès aux tableaux du régime général et du régime agricole de la Sécurité Sociale, ED 835, Mise à jour mai 2012,Paris, INRS, 360 p.

26. Saisonnalité des nouveaux cas de diabète de type 1 en Pologne. Médecine Mal Métaboliques. 1 oct 2017;11(6):567-8.

27. Geoffroy PA, Bellivier F, Scott J, Etain B. Seasonality and bipolar disorder: a systematic review, from admission rates to seasonality of symptoms. J Affect Disord. oct 2014;168:210-23.

28. Geoffroy PA, Fovet T, Micoulaud-Franchi J-A, Boudebesse C, Thomas P, Etain B, et al. Luminothérapie et épisodes dépressifs saisonniers du trouble bipolaire. 22 nov 2015 
29. ORS (Observatoire Régional de la Santé) Nord - Pas-de-Calais Centre de documentation en Promotion et Education de la Santé Lille [Internet]. [cité 29 sept 2018]. Disponible sur: http://doc.hubsante.org/index.php?lvl=publisher_see\&id=2795\#.W6-fwvk6_IU

30. Provost $\mathrm{D}$, Prouvost $\mathrm{H}$, Rivière $\mathrm{S}$, Fernet $\mathrm{F}$, Valenty $\mathrm{M}$. Les maladies à caractère professionnel (MCP) dans les métiers de l'aide et de l'assistance à domicile à travers le programme de surveillance des MCP.

31. Pinquart M, Sörensen S. Correlates of physical health of informal caregivers: a meta-analysis. J Gerontol B Psychol Sci Soc Sci. mars 2007;62(2):P126-137.

32. Redinbaugh EM, MacCallum RC, Kiecolt-Glaser JK. Recurrent syndromal depression in caregivers. Psychol Aging. sept 1995;10(3):358-68.

33. Blais C, Jean S, Sirois C, Rochette L, Plante C, Larocque I, et al. Quebec Integrated Chronic Disease Surveillance System (QICDSS), an innovative approach. Chronic Dis Inj Can. nov 2014;34(4):226-35. 
Annexes

Annexe 1 : Lettre de mission du Premier Ministre (22/01/2018) 


\section{Sog}

\section{Madame la députée,}

La qualité de vie au travail s'est affirmée comme une préoccupation croissante tant des salariés que des entreprises et est devenue un objet de négociation. Elle traduit la nécessité de ne pas opposer et de reconnaître la complémentarité entre santé et performance. Favoriser la réalisation d'un travail de qualité dans des conditions de travail satisfaisantes est un enjeu majeur de politique publique.

La qualité de vie au travail renvoie à des dimensions collectives et individuelles : le temps de travail, la charge de travail, l'organisation du travail, le climat des relations humaines, les conditions pratiques de réalisation des tâches. Une telle orientation suppose avant tout de déployer une action efficace en matière de prévention des risques professionnels, qui doit avoir pour objectif majeur la réduction des accidents du travail, encore trop nombreux, et l'amélioration des conditions de travail.

Une telle action s'inscrit dans un cadre historique : la prévention des risques professionnels et la protection des travailleurs, l'assurance contre les accidents du travail ont donné lieu historiquement aux premières lois sociales de notre pays. C'est également un domaine d'action de l'Union européenne. La construction de l'Europe sociale constitue sur ce sujet une réalité concrète avec une directive cadre et des garanties sociales nombreuses.

Sur la proposition de la ministre du travail, Madame Muriel PÉNICAUD, et de la Ministre des solidarités et de la santé, Madame Agnès BUZYN, je souhaite que la réflexion sur les voies d'amélioration de notre système de prévention des risques professionnels s'articule autour de deux axes.

Le premier axe concernera utilement la définition des enjeux et des objectifs de ce système, et son évaluation au regard de ces enjeux et objectifs. Le Président de la République et le Gouvemement ont fait de l'évaluation de l'action publique un objectif structurant de toute réflexion, venant irriguer les différentes évolutions envisagées et la mise en cuvre des orientations.

Madame Charlotte LECOCQ

Députée

Assemblée Nationale

126 , rue de l'Université

75291 PARIS CEDEX 06 
Or, il est encore difficile d'appréhender l'évolution de la qualité de vie au travail et de mesurer la performance de notre système de prévention des risques professionnels, dans le temps et dans l'espace. Cela nécessite de définir les voies et moyens pour comparer cette performance avec celle des autres systèmes existants chez nos voisins, notamment européens, qui s'inscrivent comme nous dans le respect des directives sur la protection de la santé au travail.

Les critères comparatifs et autres éléments d'évaluation doivent être suivis de manière transparente et donner lieu à débat, comme c'est le cas pour l'évolution du marché du travail. Dans ce cadre, la mise en avant de pratiques exemplaires pourra être encouragée afin de valoriser les mesures adoptées en entreprises qui favorisent la sécurité et plus encore le bienêtre au travail.

Le second axe de réflexion porte sur les leviers opérationnels à actionner pour atteindre des résultats. Ils sont multiples, et notamment juridiques et organisationnels.

Notre système français est organisé autour d'un ensemble de normes spécifiques contenues dans le code du travail, déclinant les principes généraux de prévention des risques professionnels. La réglementation et une jurisprudence croissante organisent la responsabilité de l'employeur. Le droit précise le rôle des différents acteurs du monde de l'entreprise, notamment la représentation du personnel. Mais les salariés victimes d'un accident du travail ou d'une maladie professionnelle bénéficient aussi d'un régime de protection sociale financé par les entreprises, organisé de manière à favoriser la prévention des risques professionnels. Force est de constater que l'ensemble demeure complexe.

Par ailleurs, des acteurs nombreux disposent de prérogatives parfois complémentaires, parfois similaires pour appuyer ou contrôler la prise en compte effective de l'exigence de protection des travailleurs contre les risques professionnels et de promotion de conditions de travail de qualité.

Les services de santé au travail ont fait l'objet de plusieurs réformes au cours des dernières années visant toutes à favoriser une approche pluridisciplinaire de la santé au travail, dans un contexte de désaffection préoccupante de la médecine du travail : en 10 ans, leur nombre a baissé de $30 \%$ et aujourd'hui, $75 \%$ des médecins du travail ont plus de 55 ans. Le médecin conseil des caisses de sécurité sociale intervient également dans des conditions qui peuvent parfois conduire les entreprises et les salariés à percevoir des contradictions avec la médecine du travail. C'est également le cas des différents corps de contrôle qui vérifient l'application des règles et conseillent les entreprises, salariés et leurs représentants en ce sens entre l'inspection du travail et la caisse d'assurance retraite et de la santé au travail notamment.

Enfin, différents organismes œuvrent dans le domaine de l'appui documentaire, méthodologique, de l'aide à la prévention des risques professionnels tels que l'agence nationale pour l'amélioration des conditions de travail, l'institut national de recherche et de sécurité pour la prévention des maladies professionnelles et des accidents du travail, l'organisme professionnel de prévention du bâtiment et des travaux publics.

Le plan santé au travail a pour objet de définir les priorités politiques en la matière, de tracer une perspective sur plusieurs années, de favoriser une action efficace des acteurs et de coordonner leurs actions. C'est également le cas des conventions d'objectifs et de gestion signées avec les caisses de sécurité sociales concernées. Ces documents permettent de partager avec les partenaires sociaux et les parties prenantes les orientations promues. 
Force est de constater que ce " mille feuilles », cette multitude d"acteurs et d'institutions, semblent pas ou peu coordonnés entre eux dans l'exercice de leurs missions respectives et souvent éloignés de l'entreprise et de ses salariés.

Cette multiplicité et cet éclatement des acteurs gènèrent des besoins de coordination à travers des instances en partie paritaires comme le conseil d'orientation des conditions de travail et les comités régionaux de prévention des risques professionnels.

Dans ce contexte, je souhaite que vous puissiez formuler des propositions avec Monsieur Henri FOREST et Monsieur Bruno DUPUIS visant à :

organiser très concrètement l'évaluation dans le temps et dans l'espace de la performance de notre système de prévention des risques professionnels. Vous pourrez également mener une réflexion sur la méthode permettant de valoriser les bonnes pratiques et leur permettre d'être riches d'enseignements ;

améliorer l'efficacité et l'efficience de l'ensemble du système de prévention des risques professionnels et de notre système de contrôle et d'appui au bénéfice d'une part des travailleurs, dont la protection doit être assurée et la qualité du travail et de vie au travail soutenue, et d'autre part des entreprises, dont la responsabilité doit être organisée dans un cadre sécurisé et prévisible : normes, gouvernance, organisation nationale et locale, modes d'action auprès et dans les entreprises.

Un décret vous nommera, en application des dispositions de l'article L.O.144 du code électoral, en mission auprès de Madame Muriel PÉNICAUD, Ministre du travail, et de Madame Agnès BUZYN, Ministre des solidarités et de la santé.

Vous vous appuierez à cette fin sur les services des ministères du travail et des solidarités et de la santé et procéderez à de larges consultations auprès des partenaires sociaux et des organismes de sécurité sociale. Vous étudierez également les exemples étrangers et particulièrement européens susceptibles d'éclairer utilement les constats et évolutions nécessaires.

Les conclusions de vos travaux sont attendues pour le 30 avril 2018.

Je vous prie d'agréer, Madame la Députée, l'expression de mes respectueux hommages.

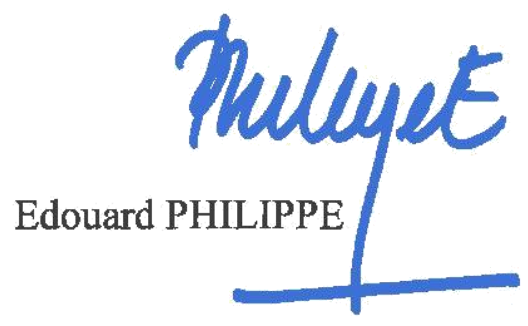




\section{Annexe 2 : Recommandations du rapport Lecocq}

Recommandation $\mathrm{n}^{\circ} 1$ : Donner davantage de visibilité nationale à la politique de santé au travail

Recommandation $\mathrm{n}^{\circ} 2$ : Consacrer un effort financier dédié et significatif à la prévention

Recommandation $n^{\circ} 3$ : Inciter les branches à s'emparer des questions de santé et de qualité de vie au travail

Recommandation $\mathrm{n}^{\circ} 4$ : Inciter les entreprises à s'engager davantage dans la prévention par une approche valorisante

Recommandation $n^{\circ} 5$ : Mieux articuler la santé au travail et la santé publique pour une meilleure prise en charge de la santé globale des travailleurs

Recommandation $n^{\circ} 6$ : Renforcer le rôle de la structure régionale et du médecin du travail pour prévenir la désinsertion professionnelle

Recommandation $n^{\circ} 7$ : Mobiliser efficacement la ressource de temps disponible des médecins du travail et des personnels de santé

Recommandation $n^{\circ} 8$ : Former les différents acteurs de la prévention dans un objectif interdisciplinaire

Recommandation $n^{\circ} 9$ : Mieux prendre en charge la prévention des risques liés aux organisations de travail et à leurs transformations

Recommandation $\mathrm{n}^{\circ} 10$ : Mettre en place au sein de chaque structure régionale une cellule spécifiquement dédiée à la prise en charge des RPS

Recommandation $n^{\circ} 11$ : Organiser au sein de la structure régionale un guichet unique

Recommandation $n^{\circ} 12$ : Permettre l'exploitation collective des données à des fins d'évaluation et de recherche et généraliser l'interopérabilité des systèmes d'information 
Recommandation $n^{\circ} 13$ : Simplifier l'évaluation des risques dans les entreprises pour la rendre opérationnelle

Recommandation $n^{\circ} 14$ : Proportionner les obligations et les moyens à déployer dans les entreprises en fonction de leur spécificité et des risques effectivement rencontrés par les salariés

Recommandation $\mathrm{n}^{\circ} 15$ : Donner les moyens aux partenaires sociaux de participer à la conception, la mise en œuvre et au suivi des politiques publiques en matière de santé au travail

Recommandation $n^{\circ} 16$ : Conduire une réflexion pour l'amélioration de la santé et de la qualité de vie au travail de la fonction publique 


\section{SERMENT}

En présence des Maîtres de cette école, de mes chers condisciples et devant ['effigie d'Fippocrate, je promets et je jure, au nom de ['Etre suprême, d'être fidèle aux lois de l'honneur et de la probité dans Cexercice de la médecine.

$>$ Je donnerai mes soins gratuits à lindigent et n'exigerai jamais un salaire au-dessus de mon travail.

Admis (e) dans l'intérieur des maisons, mes yeux ne verront pas ce qui s'y passe, ma langue taira les secrets qui me seront confiés, et mon état ne servira pas à corrompre les mours, ni à favoriser le crime.

Respectueux (se) et reconnaissant (e) envers mes Maîtres, je rendrai à leurs enfants C'instruction que j'ai reçue de leurs pères.

Que les hommes m'accordent leur estime si je suis fidèle à mes promesses. Que je sois couvert (e) d'opprobre et méprisé (e) de mes confrères sij'y manque. 


\section{Résumé}

Objectif : La veille sanitaire permet de mettre en place des actions de prévention ciblées. Cela passe par l'étude d'indicateurs objectifs en santé au travail permis par l'utilisation de thésaurus harmonisés dans les dossiers médicaux en santé au travail. Les objectifs de cette étude sont d'identifier et de décrire des groupes professionnels homogènes ayant une forte incidence des inaptitudes médicales au poste de travail.

Méthode : Une étude épidémiologique prospective a été réalisée sur une cohorte allant de 2014 à 2017 de tous les salariés suivis dans un service de santé au travail. L'extraction, le data management et l'analyse des données anonymisées ont été réalisés. L'évolution de l'incidence des inaptitudes a été observée d'abord dans la population générale, puis par secteurs d'activité et enfin par professions. Les groupes professionnels ont été comparés par analyse multivariée.

Résultats : 3614 cas d'inaptitudes ont été observées pour environ 114500 salariés suivis annuellement. L'incidence annuelle moyenne était de 7.9 inaptitudes pour mille salariés, en évolution croissante depuis 2014. Etre une femme, avoir plus de 50 ans et être en surpoids étaient plus à risque d'inaptitude. Une incidence très élevée a pu être observée chez les agents de service et aides à domicile qui semblent être une population fragile avec une tendance à l'augmentation des déclarations des troubles psychiques.

Conclusion : L'analyse des données des services de santé au travail permettrait d'identifier des populations cibles et la mise en place d'actions de prévention adaptées. Elle pourrait également aider à la prescription des périodicités de suivis. Cependant, il reste primordial de promouvoir l'harmonisation de la tenue des dossiers médicaux en santé au travail informatisés et d'élaborer un outil permettant de définir la ou les pathologies directement en cause dans les inaptitudes.

Mots-clefs : inaptitudes médicales, inaptitude médicale au poste de travail, Dossier médical en santé au travail, diagnostic territorial 